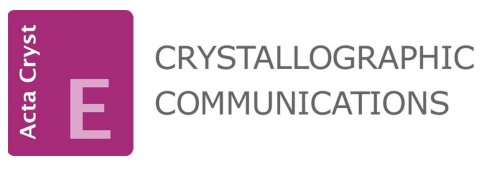

ISSN 2056-9890
Received 24 January 2020

Accepted 5 February 2020

Edited by C. Massera, Università di Parma, Italy

Keywords: crystal structure; tetrakis-substituted pyrazine; cadmium(II); zinc(II); mononuclear complexes; $\mathrm{C}-\mathrm{H} \cdots \pi$ interactions; metal-halide $\cdots \pi$ (pyrazine) contacts.

CCDC references: 1982100; 1982099

Supporting information: this article has supporting information at journals.iucr.org/e

\section{The crystal structures and Hirshfeld surface analyses of a cadmium(II) and a zinc(II) mono- nuclear complex of the new tetrakis-substituted pyrazine ligand $N, N^{\prime}, N^{\prime \prime}, N^{\prime \prime \prime}$-[pyrazine-2,3,5,6- tetrayltetrakis(methylene)]tetrakis( $N$-methylaniline)}

\author{
Ana Tesouro Vallina ${ }^{a}$ and Helen Stoeckli-Evans ${ }^{b *}$
}

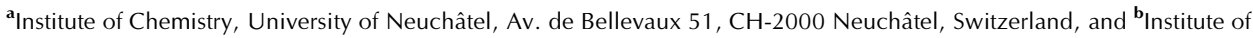
Physics, University of Neuchâtel, rue Emile-Argand 11, CH-2000 Neuchâtel, Switzerland. *Correspondence e-mail: helen.stoeckli-evans@unine.ch

The whole molecule of the cadmium(II) complex, diiodido $\left\{N, N^{\prime}, N^{\prime \prime}, N^{\prime \prime \prime}\right.$ [pyrazine-2,3,5,6-tetrayltetrakis(methylene)] tetrakis ( $N$-methylaniline)$\left.\kappa^{3} N^{2}, N^{1}, N^{6}\right\}$ cadmium(II), [ $\left.\mathrm{CdI}_{2}\left(\mathrm{C}_{36} \mathrm{H}_{40} \mathrm{~N}_{6}\right)\right]$, (I), of the ligand $N, N^{\prime}, N^{\prime \prime}, N^{\prime \prime \prime}$ [pyrazine-2,3,5,6-tetrayltetrakis(methylene)] tetrakis $(N$-methylaniline) (L), is generated by a twofold rotation symmetry; the twofold axis bisects the cadmium atom and the nitrogen atoms of the pyrazine ring. The ligand coordinates in a mono-tridentate manner and the cadmium atom has a fivefold $\mathrm{CdN}_{3} \mathrm{I}_{2}$ coordination environment with a distorted shape. In the zinc(II) complex, dichlorido $\left\{N, N^{\prime}, N^{\prime \prime}, N^{\prime \prime \prime}\right.$-[pyrazine-2,3,5,6-tetrayltetrakis(methylene) $]$ tetrakis $(N$ methylaniline)- $\kappa^{3} N^{2}, N^{1}, N^{6}$ zinc(II) dichloromethane 0.6-solvate, $\left[\mathrm{ZnCl}_{2}\left(\mathrm{C}_{36} \mathrm{H}_{40} \mathrm{~N}_{6}\right)\right] \cdot 0.6 \mathrm{CH}_{2} \mathrm{Cl}_{2}$, (II), ligand $\mathbf{L}$ also coordinates in a monotridentate manner and the zinc atom has a fivefold $\mathrm{ZnN}_{3} \mathrm{Cl}_{2}$ coordination environment with a distorted shape. It crystallized as a partial dichloromethane solvate. In the crystal of $\mathbf{I}$, the complex molecules are linked by weak $\mathrm{C}-\mathrm{H} \cdots \mathrm{I}$ contacts, forming ribbons propagating along [100]. In the crystal of II, the complex molecules are linked by a series of $\mathrm{C}-\mathrm{H} \cdots \pi$ interactions, forming layers lying parallel to the (111) plane. In the crystals of both compounds there are metal-halide $\cdots \pi$ (pyrazine) contacts present. The Hirshfeld analyses confirm the importance of the $\mathrm{C}-\mathrm{H} \cdots$ halide contacts in the crystal packing of both compounds.

\section{Chemical context}

The title ligand, $N, N^{\prime}, N^{\prime \prime}, N^{\prime \prime \prime}$-[pyrazine-2,3,5,6-tetrayltetrakis(methylene)]tetrakis $(N$-methylaniline) $(\mathbf{L})$, whose synthesis and crystal structure have been described in the preceding publication (Tesouro Vallina \& Stoeckli-Evans, 2020), is a new tetrakis-substituted pyrazine derivative. It was designed to study its coordination behaviour with transition metals (Tesouro Vallina, 2001). The reaction of the ligand with $\mathrm{CdI}_{2}$ and $\mathrm{ZnCl}_{2}$ lead to the formation of the title mononuclear complexes I and II. Herein, we describe their syntheses, molecular and crystal structures and the analyses of their Hirshfeld surfaces.

\section{Structural commentary}

The molecular structure of the cadmium(II) complex, $\mathrm{Cd}(\mathbf{L}) \mathrm{I}_{2}$ (I), of the ligand $N, N^{\prime}, N^{\prime \prime}, N^{\prime \prime \prime}$-[pyrazine-2,3,5,6-tetrayltetrakis(methylene) $]$ tetrakis $(N$-methylaniline) $(\mathbf{L})$, is illustrated in 
Fig. 1. Selected geometrical parameters are given in Table 1. The complex possesses twofold rotation symmetry, with the twofold axis bisecting the cadmium atom, Cd1, and the nitrogen atoms $\mathrm{N} 1$ and $\mathrm{N} 4$ of the pyrazine ring. The ligand coordinates in a mono-tridentate manner and the cadmium atom has a fivefold $\mathrm{CdN}_{3} \mathrm{I}_{2}$ coordination environment with a distorted shape (see Fig. $2 a$ ). The $\tau_{5}$ parameter for the fivefold coordination of atom $\mathrm{Cd} 1$ is $0.14\left(\tau_{5}=0\right.$ for a perfect squarepyramidal geometry and $=1$ for a trigonal-pyramidal geometry; Addison et al., 1984).

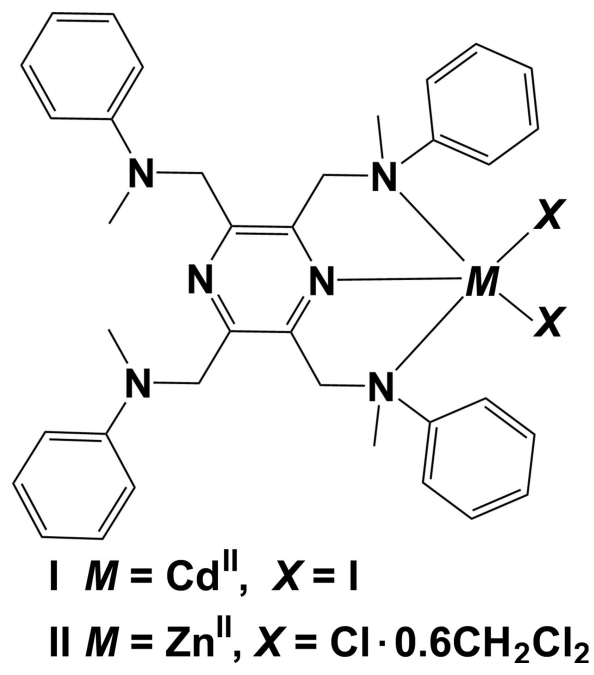

A search of the Cambridge Structural Database (CSD, Version 5.41, last update November 2019; Groom et al., 2016) for a $\mathrm{CdN}_{3} \mathrm{I}_{2}$ coordination environment involving a pyrazine $\mathrm{N}$ atom yielded only one relevant structure, the $\mathrm{CdI}_{2}$ mononuclear complex of the ligand 2,3,5,6-tetrakis(pyridin-2-

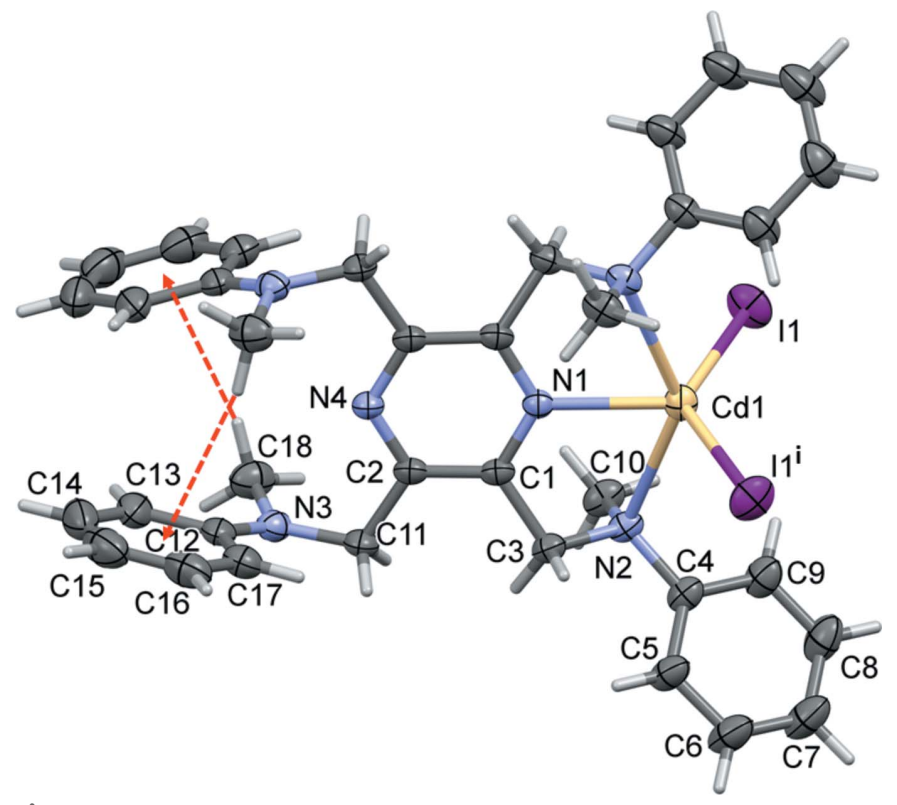

Figure 1

A view of the molecular structure of complex $\mathbf{I}$, with atom labelling [symmetry code (i): $-x+\frac{3}{2}, y,-z$ ]. Displacement ellipsoids are drawn at the $30 \%$ probability level. The intramolecular $\mathrm{C}-\mathrm{H} \cdots \pi$ interactions are shown as dashed red arrows (Table 2).
Table 1

Selected geometric parameters $\left(\AA,^{\circ}\right)$ for $\mathbf{I}$.

\begin{tabular}{lclr}
\hline $\mathrm{Cd} 1-\mathrm{N} 1$ & $2.295(3)$ & $\mathrm{Cd} 1-\mathrm{I} 1$ & $2.7038(3)$ \\
$\mathrm{Cd} 1-\mathrm{N} 2$ & $2.599(3)$ & & \\
& & & \\
$\mathrm{N} 1-\mathrm{Cd} 1-\mathrm{N} 2$ & $69.65(6)$ & $\mathrm{N} 2-\mathrm{Cd} 1-\mathrm{I} 1^{\mathrm{i}}$ & $95.35(6)$ \\
$\mathrm{N} 22^{\mathrm{i}}-\mathrm{Cd} 1-\mathrm{N} 2$ & $139.31(12)$ & $\mathrm{N} 2-\mathrm{Cd} 1-\mathrm{I} 1$ & $101.29(6)$ \\
$\mathrm{N} 1-\mathrm{Cd} 1-\mathrm{I} 1$ & $114.551(10)$ & $\mathrm{I}^{\mathrm{i}}-\mathrm{Cd} 1-\mathrm{I} 1$ & $130.90(2)$ \\
\hline
\end{tabular}

Symmetry code: (i) $-x+\frac{3}{2}, y,-z$.

Table 2

Hydrogen-bond geometry $\left(\AA,^{\circ}\right)$ for $\mathbf{I}$.

$\mathrm{Cg} 3$ is the centroid of the pyrazine ring $\mathrm{N} 1 / \mathrm{N} 4 / \mathrm{C} 1 / \mathrm{C} 2 / \mathrm{Cl}^{i} / \mathrm{C}^{\mathrm{i}}$ and $\mathrm{Cg} 5$ is the centroid of the $\mathrm{C} 12-\mathrm{C} 17$ ring.

\begin{tabular}{lllll}
\hline$D-\mathrm{H} \cdots A$ & $D-\mathrm{H}$ & $\mathrm{H} \cdots A$ & $D \cdots A$ & $D-\mathrm{H} \cdots A$ \\
\hline $\mathrm{C} 18-\mathrm{H} 18 C \cdots \mathrm{Cg} 5^{\text {i }}$ & 0.97 & 2.95 & $3.896(5)$ & 165 \\
$\mathrm{C} 17-\mathrm{H} 17 \cdots \mathrm{I} 1^{\text {ii }}$ & 0.94 & 3.09 & $3.907(4)$ & 147 \\
$\mathrm{Cd} 1-\mathrm{I} 1 \cdots \mathrm{Cg}^{\text {iii }}$ & $2.70(1)$ & $3.96(1)$ & $6.5131(12)$ & $155(1)$ \\
$\mathrm{Cd} 1-\mathrm{I} 1 \cdots \mathrm{Cg} 3^{\text {iv }}$ & $2.70(1)$ & $3.96(1)$ & $6.5131(12)$ & $155(1)$ \\
\hline
\end{tabular}

Symmetry codes: (i) $-x+\frac{3}{2}, y,-z$; (ii) $x+\frac{1}{2},-y, z$; (iii) $-x+1,-y,-z$; (iv) $x-\frac{1}{2},-y, z$.

yl)pyrazine (TPPZ), viz. complex (2,3,5,6-tetrakis(pyridin-2yl)pyrazine)bis(iodo)cadmium(II) (GAHRIT; Saghatforoush, 2015). Here the $\tau_{5}$ parameter for the cadmium atom is 0.04 . The $\mathrm{Cd}-\mathrm{N}_{\mathrm{pz}}$ bond length is $c a 2.388 \AA$ compared to 2.295 (3) $\AA$ in I, while the Cd-I bond lengths are ca 2.741 and $2.727 \AA$ compared to 2.7038 (3) $\AA$ in I. The N-methylaniline groups on the non-coordinated side of the ligand are linked by intramolecular $\mathrm{C}-\mathrm{H} \cdots \pi$ interactions (Fig. 1 and Table 2).

The molecular structure of the zinc(II) complex, $\mathrm{Zn}(\mathbf{L}) \mathrm{Cl}_{2} \cdot 0.6\left(\mathrm{CH}_{2} \mathrm{Cl}_{2}\right)$ (II), is illustrated in Fig. 3. It crystallized as a partial dichloromethane solvate. Selected geometrical parameters are given in Table 3. The ligand $\mathbf{L}$ coordinates in a mono-tridentate manner and the zinc atom, $\mathrm{Zn} 1$, has a fivefold $\mathrm{ZnN}_{3} \mathrm{Cl}_{2}$ coordination environment with a distorted shape (see Fig. $2 b$ ). The $\tau_{5}$ parameter for atom $\mathrm{Zn} 1$ is 0.30 .

A search of the $\mathrm{CSD}$ for a $\mathrm{ZnN}_{3} \mathrm{Cl}_{2}$ coordination environment involving a pyrazine $\mathrm{N}$ atom yielded five relevant structures, which again involve the ligand TPPZ. They include two polymorphs of the mononuclear complex dichloro$\left[2,3,5,6\right.$-tetrakis(2-pyridyl)pyrazine- $\left.N, N^{\prime}, N^{\prime \prime}\right]$ zinc(II): a mono-
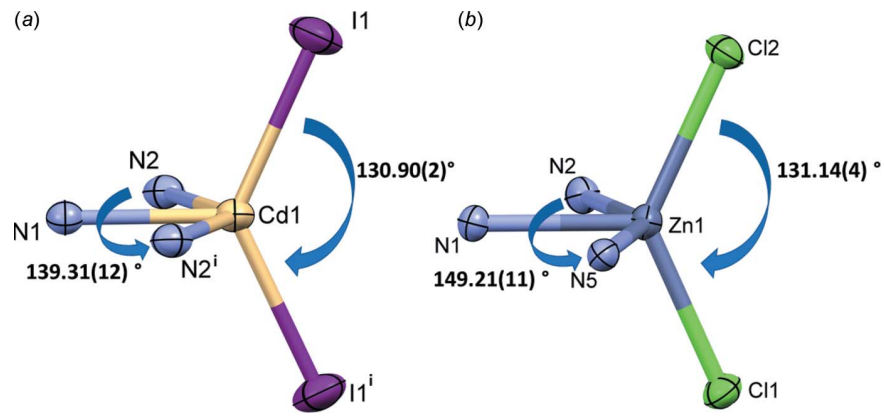

Figure 2

A comparison of the coordination spheres of $(a)$ the cadmium atom in complex I [symmetry code (i): $\left.-x+\frac{3}{2}, y,-z\right]$, and $(b)$ the zinc atom in complex II. 
Table 3

Selected geometric parameters $\left(\AA,^{\circ}\right)$ for $\mathbf{I I}$.

\begin{tabular}{lclr}
\hline $\mathrm{Zn} 1-\mathrm{N} 1$ & $2.057(3)$ & $\mathrm{Zn} 1-\mathrm{Cl} 1$ & $2.2251(10)$ \\
$\mathrm{Zn} 1-\mathrm{N} 2$ & $2.385(3)$ & $\mathrm{Zn} 1-\mathrm{Cl} 2$ & $2.2425(11)$ \\
$\mathrm{Zn} 1-\mathrm{N} 5$ & $2.413(3)$ & & \\
& & & \\
$\mathrm{N} 1-\mathrm{Zn} 1-\mathrm{N} 2$ & $75.02(12)$ & $\mathrm{Cl} 1-\mathrm{Zn} 1-\mathrm{N} 2$ & $98.12(8)$ \\
$\mathrm{N} 1-\mathrm{Zn} 1-\mathrm{N} 5$ & $74.23(12)$ & $\mathrm{Cl} 2-\mathrm{Zn} 1-\mathrm{N} 2$ & $95.70(9)$ \\
$\mathrm{N} 2-\mathrm{Zn} 1-\mathrm{N} 5$ & $149.21(11)$ & $\mathrm{Cl} 1-\mathrm{Zn} 1-\mathrm{N} 5$ & $93.02(7)$ \\
$\mathrm{N} 1-\mathrm{Zn} 1-\mathrm{Cl} 1$ & $114.15(9)$ & $\mathrm{Cl} 2-\mathrm{Zn} 1-\mathrm{N} 5$ & $98.34(8)$ \\
$\mathrm{N} 1-\mathrm{Zn} 1-\mathrm{C} 2$ & $114.68(9)$ & $\mathrm{Cl} 1-\mathrm{Zn} 1-\mathrm{Cl} 2$ & $131.14(4)$ \\
\hline
\end{tabular}

Table 4

Hydrogen-bond geometry $\left(\AA,^{\circ}\right)$ for II.

$\mathrm{Cg} 3$ is the centroid of the pyrazine ring N1/N4/C1/C2/C21/C22, and $\mathrm{Cg} 5$ and $\mathrm{Cg} 7$ are the centroids of rings $\mathrm{C} 12-\mathrm{C} 17$ and $\mathrm{C} 32-\mathrm{C} 37$, respectively.

\begin{tabular}{lllll}
\hline$D-\mathrm{H} \cdots A$ & $D-\mathrm{H}$ & $\mathrm{H} \cdots A$ & $D \cdots A$ & $D-\mathrm{H} \cdots A$ \\
\hline $\mathrm{C} 6-\mathrm{H} 6 \cdots \mathrm{Cg} 7^{\mathrm{i}}$ & 0.94 & 2.88 & $3.814(6)$ & 177 \\
$\mathrm{C} 11-\mathrm{H} 11 B \cdots \mathrm{Cg} 5^{\text {ii }}$ & 0.98 & 2.90 & $3.540(5)$ & 124 \\
$\mathrm{C} 26-\mathrm{H} 26 \cdots \mathrm{Cg} 3^{\text {iii }}$ & 0.94 & 2.95 & $3.544(5)$ & 122 \\
$\mathrm{Zn} 1-\mathrm{C} 2 \cdots \cdots 3^{\text {iv }}$ & $2.24(1)$ & $3.68(1)$ & $5.8035(19)$ & $156(1)$ \\
\hline
\end{tabular}

Symmetry codes: (i) $x-1, y-1, z$; (ii) $-x+1,-y,-z$; (iii) $-x+1,-y+1,-z+1$; (iv) $-x+1,-y,-z+1$.

clinic polymorph (WAGPOJ; Graf et al., 1993) and a triclinic polymorph (WAGPOJ01; Saljooghi \& Fatemi, 2011). There are two structures of the binuclear complex $\left[\mu_{2}-2,3,5,6\right.$-tetrakis(2-pyridyl)pyrazine]tetrachlorodizinc(II): one hydrated (DOMHOD; Trivedi et al., 2009), the other not (PAPCER; Hong et al., 2017), and finally, the unusual polynuclear complex octakis $\left(\mu_{2}\right.$-chloro)bis[ $\mu_{2}-2,3,5,6$-tetrakis(2-pyridyl)pyrazine]dodecachlorotetraaquadecazinc (WIBVOS; Graf $\&$ Stoeckli-Evans, 1994). For these five structures, the $\tau_{5}$ parameter for the zinc atoms varies from 0.08 in WAGPOJ to 0.36 in WAGPOJ01. The latter is similar to the value of 0.30 for II. The $\mathrm{Zn}-\mathrm{N}_{\mathrm{pz}}$ bond lengths vary from $c a 2.141$ to $2.200 \AA$ compared to 2.057 (3) $\AA$ in II, while the $\mathrm{Zn}-\mathrm{Cl}$ bond lengths vary from $c a 2.232$ to $2.343 \AA$ compared to 2.2251 (10) and 2.2425 (11) $\AA$ in II.

The conformation of the ligand $\mathbf{L}$ differs in the two complexes (Fig. 4). The orientation of the phenyl rings with respect to the pyrazine ring and to each other is slightly

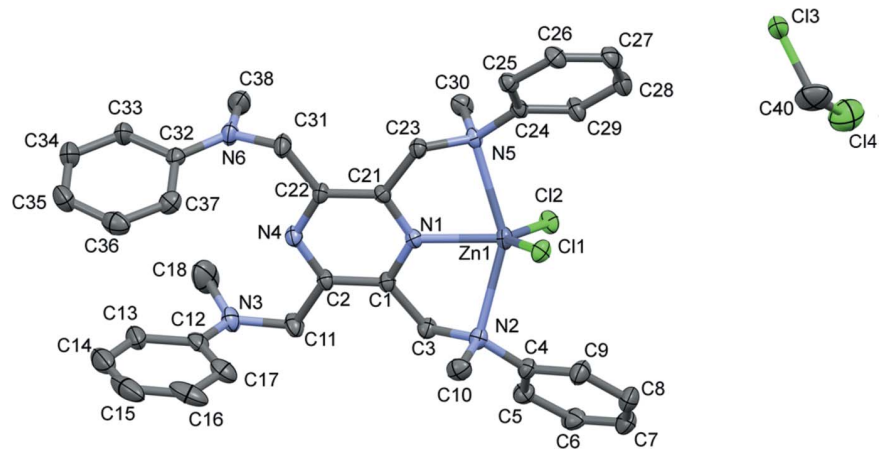

Figure 3

A view of the molecular structure of compound II, with atom labelling. Displacement ellipsoids are drawn at the $30 \%$ probability level. For clarity, $\mathrm{H}$ atoms have been omitted.
Table 5

A comparison of the conformation of the ligand $(\mathbf{L})$ in complexes $\mathbf{I}$ and $\mathbf{I I}$.

The definitions of rings $A, B, C, D$ and $E$ are given in Fig. 4.

\begin{tabular}{llll}
\hline Dihedral angle $\left(^{\circ}\right)$ & $\mathbf{I}^{a}$ & II & $\Delta(\mathbf{I}-\mathbf{I I})^{\circ}$ \\
\hline$A$ to $B$ & $41.9(2)$ & $35.5(2)$ & $>6.4$ \\
$A$ to $C$ & $86.1(2)$ & $87.5(3)$ & $<1.4$ \\
$A$ to $D$ & $41.9(2)$ & $34.9(2)$ & $>7.0$ \\
$A$ to $E$ & $86.1(2)$ & $74.4(2)$ & $>11.7$ \\
$B$ to $C$ & $54.0(2)$ & $53.7(3)$ & $>0.3$ \\
$B$ to $D$ & $38.0(2)$ & $26.9(2)$ & $>11.1$ \\
$B$ to $E$ & $63.4(2)$ & $71.9(2)$ & $<8.5$ \\
$C$ to $D$ & $63.4(2)$ & $58.5(3)$ & $>4.9$ \\
$C$ to $E$ & $24.9(2)$ & $18.3(3)$ & $>6.6$ \\
$D$ to $E$ & $54.0(2)$ & $74.9(2)$ & $<20.9$ \\
\hline
\end{tabular}

Note: (a) $D=B^{\text {i }}, E=C^{\text {i }}$; symmetry code: (i) $-x+\frac{3}{2}, y,-z$.

different, and the various dihedral angles are compared in Table 5. It can be seen that the most significant difference, of $20.9(2)^{\circ}$, involves the orientation of $\operatorname{ring} D\left(\operatorname{ring} B^{\mathrm{i}}\right.$ in $\left.\mathbf{I}\right)$ with respect to ring $E$ (ring $C^{\mathrm{i}}$ in $\left.\mathbf{I}\right)$.

\section{Supramolecular features}

A partial view of the crystal packing of $\mathbf{I}$ is shown in Fig. 5. Molecules are linked by weak $\mathrm{C}-\mathrm{H}$...I contacts, forming ribbons propagating along [100]; see Table 2. There are $\mathrm{Cd}-$
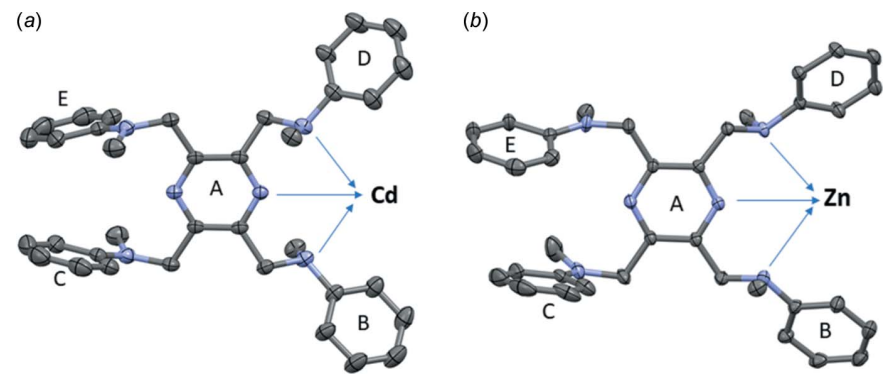

Figure 4

A comparison of the conformation of the ligand $\mathbf{L}$ in complexes $\mathbf{I}$ and $\mathbf{I I}$. For complex I, which possesses twofold rotation symmetry, ring $D=B^{\mathrm{i}}$, and ring $E=C^{\mathrm{i}}$ [symmetry code: (i) $\left.-x+\frac{3}{2}, y,-z\right]$.

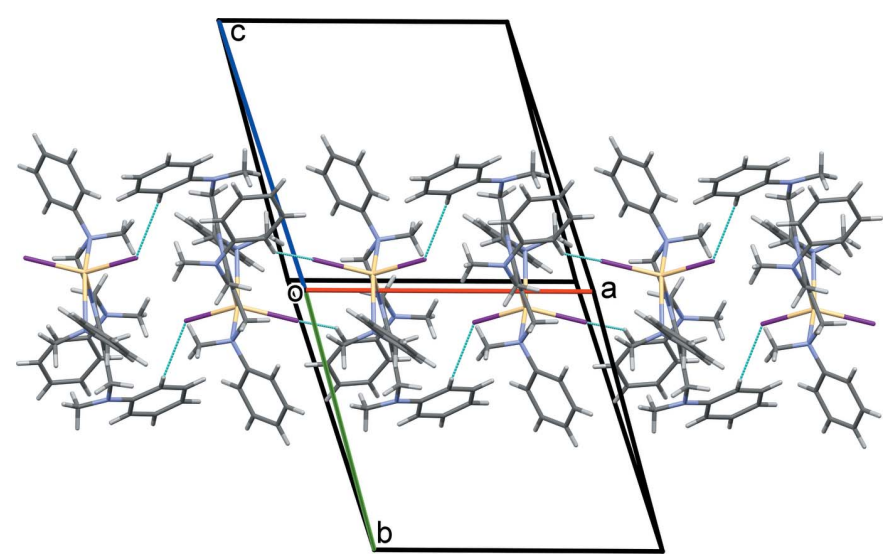

Figure 5

A view normal to plane (011) of the crystal packing of complex I. The weak $\mathrm{C}-\mathrm{H} \cdots \mathrm{I}$ interactions are shown as dashed lines (Table 2). 

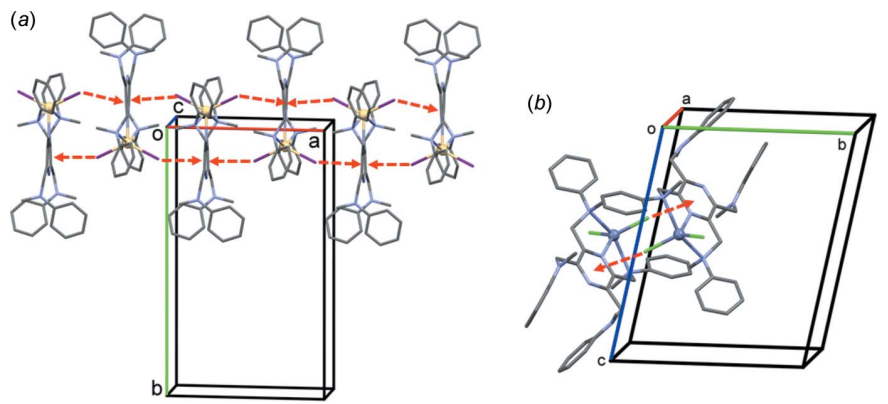

Figure 6

(a) A partial view along the $c$ axis of the crystal packing of $\mathbf{I}$, showing the Cd-I $\cdots \pi$ (pyrazine) interactions (Table 2; dashed red arrows), (b) a partial view along the $a$ axis of the crystal packing of II, showing the $\mathrm{Zn}-$ $\mathrm{Cl} \cdots \pi$ (pyrazine) interactions (Table 4; dashed red arrows). For clarity, the dichloromethane molecule has been omitted.

I $\cdots \pi$ (pyrazine) contacts present, consolidating the chains propagating along the $a$-axis direction (Fig. $6 a$ and Table 2). This situation is similar to that observed in the crystal of the $\mathrm{CdI}_{2}$ complex of TPPZ (GAHRIT; Saghatforoush, 2015). There, the I . - centroid(pyrazine ring) distance is 3.699 (1) $\AA$ with a $\mathrm{Cd}-\mathrm{I} \cdots$ centroid angle of $175.92(12)^{\circ}$, compared to $3.9593(12) \AA$ and $155.19(3)^{\circ}$ in complex I (Fig. $6 a$ and Table 2).

In the crystal of II, molecules are linked by a series of C$\mathrm{H} \cdots \pi$ interactions, forming layers lying parallel to the (111) plane; see Fig. 7 and Table 4. The dichloromethane molecules are linked across a center of symmetry with a short $\mathrm{Cl} 4 \cdots \mathrm{Cl} 4(-x,-y,-\mathrm{z}+2)$ contact of $3.045(5) \AA$ and do not participate in any significant intermolecular interactions with the complex molecule. There are $\mathrm{Zn}-\mathrm{Cl} \cdots \pi$ (pyrazine) contacts present, which link inversion-related molecules, forming dimers (Fig. $6 b$ and Table 5). This arrangement is similar to that observed in the crystal structure of the $\mathrm{ZnCl}_{2}$ complex of TPPZ (PAPCER; Hong et al., 2017). This compound crystallized with two independent molecules in the

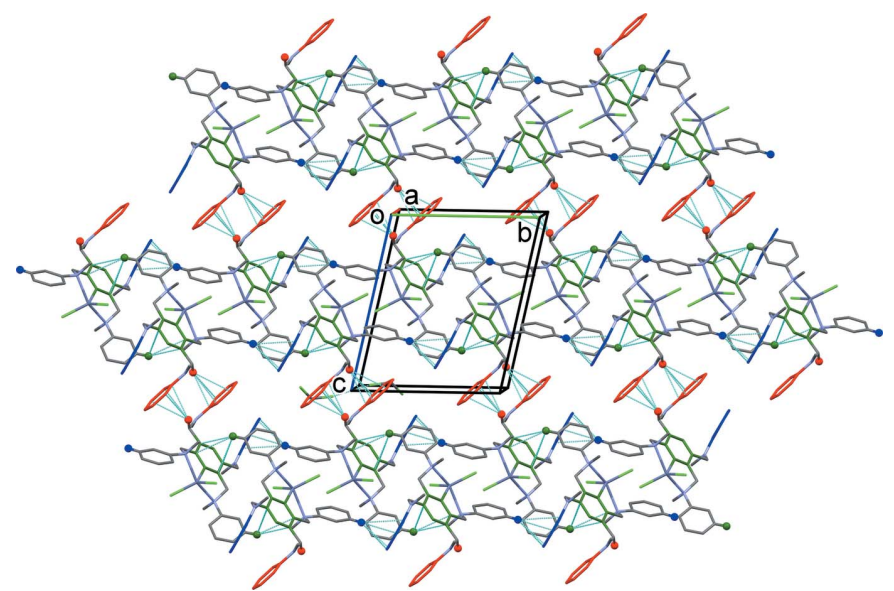

Figure 7

A view along the $a$ axis of the crystal packing of compound II. The various $\mathrm{C}-\mathrm{H} \cdots \pi$ interactions (Table 4; blue, red and green) are shown as dashed lines. The dichloromethane molecule has been omitted, and only the $\mathrm{H}$ atoms (blue, red and green) involved in the $\mathrm{C}-\mathrm{H} \cdots \pi$ interactions have been included.
Table 6

Summary of interatomic contacts $(\AA)^{a}$, shorter than the sum of the van der Waals radii, in the crystal structures of $\mathbf{I}$ and $\mathbf{I I}$.

\begin{tabular}{llll}
\hline Contact & Length & Length - vdW & Symmetry operation \\
\hline I & & & \\
$\mathrm{C} 11 \cdots \mathrm{C} 12$ & 3.278 & -0.122 & $\frac{3}{2}-x,-\frac{1}{2}-y,-\frac{1}{2}-z$ \\
$\mathrm{C} 12 \cdots \mathrm{H} 11 A$ & 2.805 & -0.095 & $\frac{3}{2}-x,-\frac{1}{2}-y,-\frac{1}{2}-z$ \\
$\mathrm{I} 1 \cdots \mathrm{H} 17$ & 3.087 & -0.093 & $-\frac{1}{2}+x,-y, z$ \\
$\mathrm{~N} 3 \cdots \mathrm{H} 11 B$ & 2.671 & -0.079 & $\frac{3}{2}-x,-\frac{1}{2}-y,-\frac{1}{2}-z$ \\
$\mathrm{~N} 3 \cdots \mathrm{C} 11$ & 3.234 & -0.016 & $\frac{3}{2}-x,-\frac{1}{2}-y,-\frac{1}{2}-z$ \\
& & & \\
II & & & $-x,-y, 2-z$ \\
$\mathrm{C} 14 \cdots \mathrm{Cl} 4$ & 3.045 & -0.455 & $-x,-y, 1-z$ \\
$\mathrm{C} 6 \cdots \mathrm{H} 40 B$ & 2.758 & -0.142 & $1-x,-y, 1-z$ \\
$\mathrm{C} 30 \cdots \mathrm{H} 3 B$ & 2.779 & -0.121 & $1-x, 1-y, 1-z$ \\
$\mathrm{H} 23 B \cdots \mathrm{H} 23 B$ & 2.287 & -0.113 & $-1+x,-1+y, z$ \\
$\mathrm{H} 6 \cdots \mathrm{C} 36$ & 2.798 & -0.102 & $-1+x, y, z$ \\
$\mathrm{Cl} 1 \cdots \mathrm{H} 33$ & 2.854 & -0.096 & $-1+x,-1+y, z$ \\
$\mathrm{H} 6 \cdots \mathrm{C} 37$ & 2.858 & -0.042 & $1-x,-y, 1-z$ \\
$\mathrm{H} 3 B \cdots \mathrm{H} 30 A$ & 2.359 & -0.041 & $1-x, 1-y, 1-z$ \\
$\mathrm{H} 10 B \cdots \mathrm{H} 26$ & 2.382 & -0.018 &
\end{tabular}

Note: (a) distances were calculated using Mercury (Macrae et al., 2008).

asymmetric unit. There, the $\mathrm{Cl}$...centroid(pyrazine ring) distances are $c a 3.087$ and $3.167 \AA$, with the corresponding $\mathrm{Zn}-\mathrm{Cl} \cdots$ centroid angles being $\mathrm{ca} 152.62$ and $141.76^{\circ}$. In the crystal structure of WIBVOS, a similar interaction is present with a $\mathrm{Cl}$ - - centroid(pyrazine ring) distance of $c a 3.987 \AA$ and a $\mathrm{Zn}-\mathrm{Cl} \cdots$ centroid angle of $c a .170 .96^{\circ}$. In complex II, the corresponding $\mathrm{Cl} \cdots$ centroid(pyrazine ring) distance and $\mathrm{Zn}-$ $\mathrm{Cl}$. centroid angle are $3.683(2) \AA$ and $155.96(6)^{\circ}$, respectively (Table 4).

\section{Hirshfeld surface analysis and two-dimensional fingerprint plots}

The Hirshfeld surface analysis (Spackman \& Jayatilaka, 2009) and the associated two-dimensional fingerprint plots (McKinnon et al., 2007) were performed with CrystalExplorer17 (Turner et al., 2017). The Hirshfeld surfaces are colour-mapped with the normalized contact distance, $d_{\text {norm }}$, ranging from red (distances shorter than the sum of the van

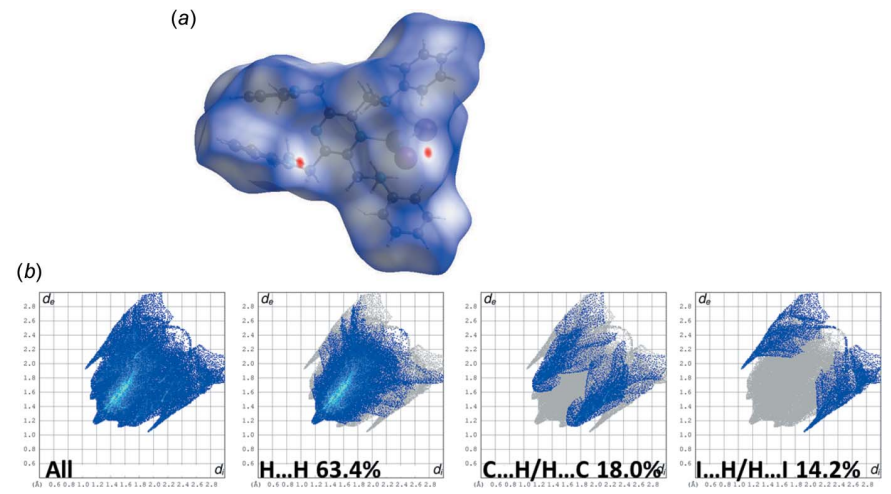

Figure 8

(a) The Hirshfeld surface of complex I, mapped over $d_{\text {norm }}$, in the colour range -0.0713 to 1.5380 a.u., (b) the full two-dimensional fingerprint plot for complex I, and fingerprint plots delineated into $\mathrm{H} \cdots \mathrm{H}, \mathrm{C} \cdots \mathrm{H} / \mathrm{H} \cdots \mathrm{C}$ and $\mathrm{I} \cdots \mathrm{H} / \mathrm{H} \cdots \mathrm{I}$ contacts. 

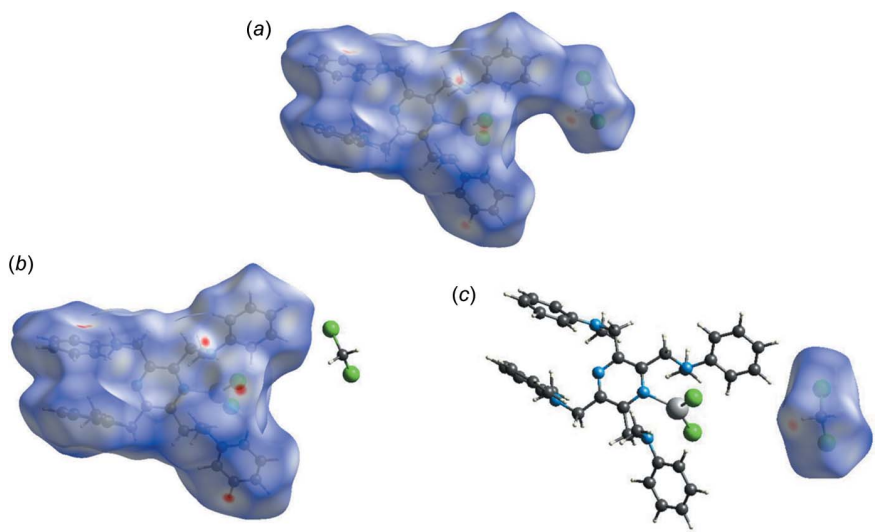

Figure 9

(a) The Hirshfeld surface of compound II, mapped over $d_{\text {norm }}$, in the colour range -0.2597 to 1.5438 a.u., $(b)$ the Hirshfeld surface of complex II, mapped over $d_{\text {norm }}$, in the colour range -0.0933 to 1.5453 a.u., (c) the Hirshfeld surface of the solvent molecule, mapped over $d_{\text {norm }}$, in the colour range -0.2602 to 1.4344 a.u..

der Waals radii) through white to blue (distances longer than the sum of the van der Waals radii). A summary of the short intermolecular contacts in the crystal structures of $\mathbf{I}$ and $\mathbf{I I}$ is given in Table 6.

For complex I, the Hirshfeld surface (HS) mapped over $d_{\text {norm }}$, and the two-dimensional fingerprint plots are given in Fig. 8. The red spots on the HS (Fig. $8 a$ ) correspond to the I $\cdots H$ contacts, which give a pair of spikes in the fingerprint plot (Fig. $8 b$ ) at $d_{\mathrm{e}}+d_{\mathrm{i}} \simeq 3.0 \AA$, contributing $14.2 \%$ to the HS. The H. .H contacts contribute $63.4 \%$ and the C..H contacts $18.0 \%$. Any other atom-atom contacts contributed less than $2 \%$ and have not been included here.
For compound II, the Hirshfeld surface mapped over $d_{\text {norm }}$, is shown in Fig. 9a, and that for the complex itself and the solvent molecule in Figs. $9 b$ and $9 c$, respectively. The faint red spots correspond to the $\mathrm{Cl} \cdot \mathrm{H}$ contacts in the crystal. These give a pair of spikes in the fingerprint plots, at $d_{\mathrm{e}}+d_{\mathrm{i}} \simeq 2.7 \AA$, contributing $22.7 \%$, in the compound (Fig. 10a) and at $d_{\mathrm{e}}+d_{\mathrm{i}}$ $\simeq 2.7 \AA$, contributing $18.1 \%$, in the complex (Fig. 10b). For the solvent molecule, a single sharp spike is observed $\left(d_{\mathrm{e}}+d_{\mathrm{i}} \simeq\right.$ $2.8 \AA$ ) with a contribution of $59.6 \%$ to the HS (Fig. 10c). The $\mathrm{H} \cdots \mathrm{H}$ contacts contribute $55.1,59.4$ and $25.2 \%$ to the Hirshfeld surfaces of the compound, the complex and the solvent molecule, respectively, while the $\mathrm{C} \cdot \mathrm{H}$ contributions are $17.7,18.8$ and $6.8 \%$, respectively. Any other atom-atom contacts contributed less than $2 \%$ and have not been included here.

\section{Synthesis and crystallization}

The synthesis and crystal structure of the ligand, $N, N^{\prime}, N^{\prime \prime}, N^{\prime \prime \prime}$ [pyrazine-2,3,5,6-tetrayltetrakis(methylene)] tetrakis $(N$-methylaniline) $\mathbf{L}$, have been described in the preceding publication (Tesouro Vallina \& Stoeckli-Evans, 2020).

Synthesis of the complex [Cd(L) $\left.\mathbf{I}_{2}\right](\mathrm{I}):$

About $10 \mathrm{ml}$ of a very dilute $\mathrm{CH}_{2} \mathrm{Cl}_{2}$ solution of ligand $\mathbf{L}$ were introduced into a glass tube and layered with $c a 2 \mathrm{ml}$ of $\mathrm{MeOH}$ as a buffer zone. Then, $10 \mathrm{ml}$ of a dilute methanolic solution of $\mathrm{CdI}_{2}$ were added slowly to avoid possible mixing. The glass tube was sealed and left at room temperature. The colour of the interphase changed immediately to deep yellow and in hours to green. After a few days, green rod-like crystals were formed. IR (KBr pellet, $\mathrm{cm}^{-1}$ ): $2922(\mathrm{~m}), 1599(\mathrm{vs}), 1507$
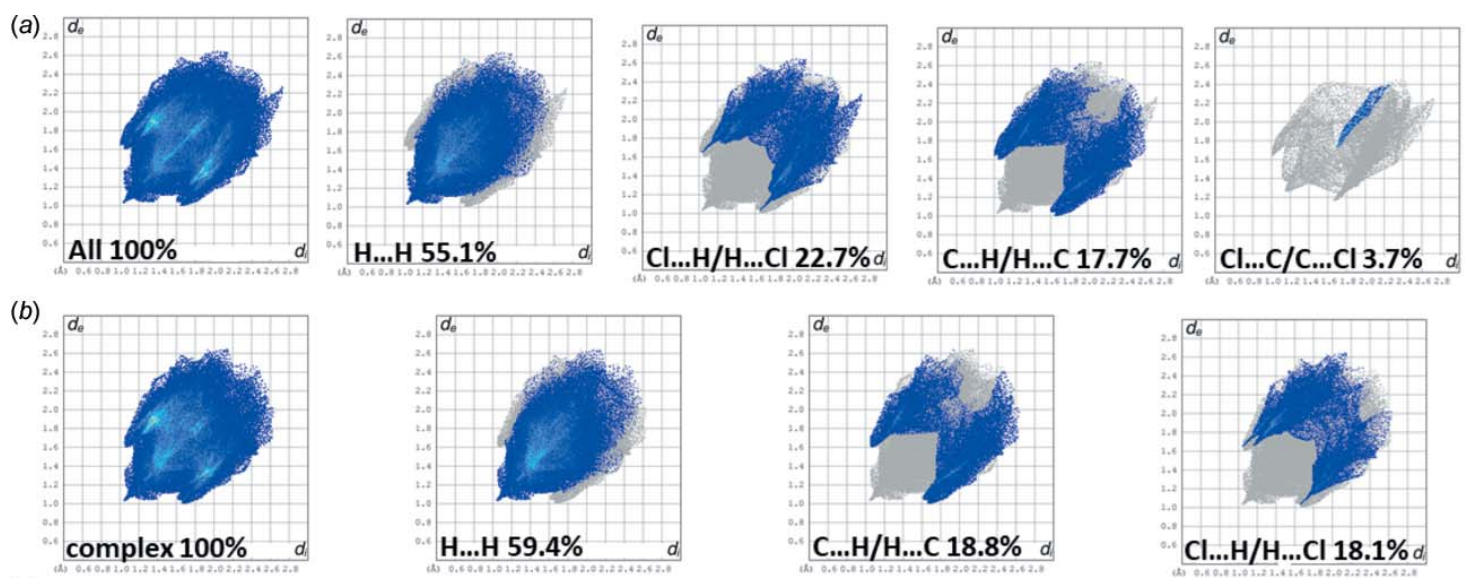

(c)
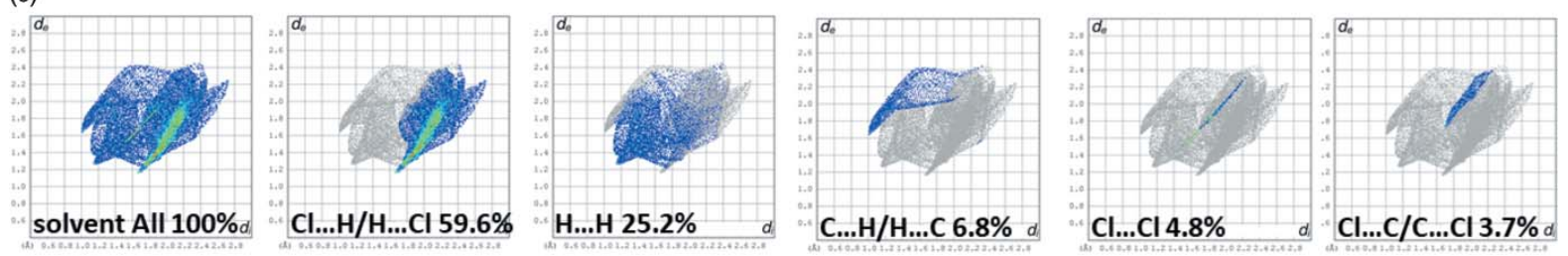

Figure 10

(a) The full two-dimensional fingerprint plot for compound II, and fingerprint plots delineated into $\mathrm{H} \cdots \mathrm{H}, \mathrm{Cl} \cdots \mathrm{H} / \mathrm{H} \cdots \mathrm{Cl}, \mathrm{C} \cdots \mathrm{H} / \mathrm{H} \cdots \mathrm{C}$ and $\mathrm{Cl} \cdots \mathrm{Cl}$

$\mathrm{C} \cdots \mathrm{Cl}$ contacts, $(b)$ the full two-dimensional fingerprint plot for complex II, and fingerprint plots delineated into $\mathrm{H} \cdots \mathrm{H}, \mathrm{C} \cdots \mathrm{H} / \mathrm{H} \cdots \mathrm{C}$ and $\mathrm{Cl} \cdots \mathrm{H} /$

$\mathrm{H} \cdots \mathrm{Cl}$ contacts, $(c)$ the full two-dimensional fingerprint plot for the solvent molecule and fingerprint plots delineated into $\mathrm{Cl} \cdots \mathrm{H} / \mathrm{H} \cdots \mathrm{Cl}, \mathrm{H} \cdots \mathrm{H}, \mathrm{C} \cdots \mathrm{H} /$ $\mathrm{H} \cdots \mathrm{C}, \mathrm{Cl} \cdots \mathrm{Cl}$ and $\mathrm{Cl} \cdots \mathrm{C} / \mathrm{C} \cdots \mathrm{Cl}$ contacts. 
Table 7

Experimental details.

I

Crystal data

Chemical formula

$M_{\text {r }}$

Crystal system, space group

Temperature (K)

$a, b, c(\AA)$

$\alpha, \beta, \gamma\left({ }^{\circ}\right)$

$V\left(\AA^{3}\right)$

Z

Radiation type

$\mu\left(\mathrm{mm}^{-1}\right)$

Crystal size (mm)

Data collection

Diffractometer

Absorption correction

$T_{\min }, T_{\max }$

No. of measured, independent and

observed $[I>2 \sigma(I)]$ reflections

$R_{\text {int }}$

$(\sin \theta / \lambda)_{\max }\left(\AA^{-1}\right)$

Refinement

$R\left[F^{2}>2 \sigma\left(F^{2}\right)\right], w R\left(F^{2}\right), S$

No. of reflections

No. of parameters

$\mathrm{H}$-atom treatment

$\Delta \rho_{\max }, \Delta \rho_{\min }\left(\mathrm{e} \AA^{-3}\right)$

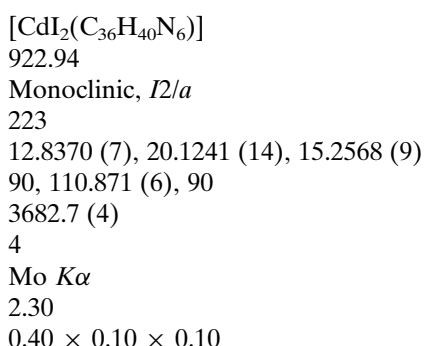

$\left[\mathrm{ZnCl}_{2}\left(\mathrm{C}_{36} \mathrm{H}_{40} \mathrm{~N}_{6}\right)\right] \cdot 0.6 \mathrm{CH}_{2} \mathrm{Cl}_{2}$

743.99

Triclinic, $P \overline{1}$

223

11.9196 (8), $12.1208(8), 13.919(1)$

98.222 (8), $100.313(8), 107.580$ (7)

1843.9 (2)

Mo $K \alpha$

0.93

$0.30 \times 0.10 \times 0.10$

STOE IPDS 1

Multi-scan (MULABS; Spek, 2020) Multi-scan (MULABS; Spek, 2020)

$0.961,1.000$

$14308,3566,2549$

0.031

0.615

$0.029,0.072,0.95$
3566
207
H-atom parameters constrained
$1.15,-0.88$

0.615

STOE IPDS 1

$0.983,1.000$

$14512,6654,3490$

$0.043,0.117,0.79$

6654

437

$\mathrm{H}$-atom parameters constrained $0.75,-0.35$

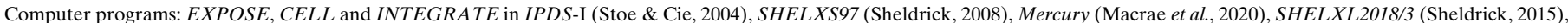
PLATON (Spek, 2020) and publCIF (Westrip, 2010).

$(s), 1497(s), 1173(m), 1120(m), 751(s), 694(s)$. No elemental analytical data are available.

\section{Synthesis of the complex $\left[\mathrm{Zn}(\mathrm{L}) \mathrm{Cl}_{2}\right] \cdot 0.6\left(\mathrm{CH}_{2} \mathrm{Cl}_{2}\right)$ (II):}

To a solution of $\mathrm{ZnCl}_{2}(0.1 \mathrm{mmol}, 0.014 \mathrm{~g})$ in $5 \mathrm{ml}$ of $\mathrm{MeOH}$, a solution of $\mathbf{L}\left(0.05 \mathrm{mmol}, 0.028 \mathrm{~g}, 5 \mathrm{ml} \mathrm{CH}_{2} \mathrm{Cl}_{2}\right)$ was added. The solution was stirred at RT for $2 \mathrm{~h}$ without any significant colour change. The clear light-green solution obtained was filtered to avoid any impurity and allowed to evaporate slowly. After a few days, yellow rod-like crystals were obtained. IR (KBr pellet, $\mathrm{cm}^{-1}$ ): 1599 (vs), $1507(\mathrm{~s}), 1451(\mathrm{~m}), 1363(\mathrm{~s}), 1257$ $(m), \quad 1171 \quad(m), \quad 1033 \quad(m), \quad 920 \quad(m), \quad 746 \quad(s)$, 691 (s). Analysis for $\left[\mathrm{Zn}\left(\mathrm{C}_{36} \mathrm{H}_{40} \mathrm{~N}_{6}\right) \mathrm{Cl}_{2}\right] \cdot 0.6 \mathrm{CH}_{2} \mathrm{Cl}_{2}$ (743.99 $\mathrm{g} \mathrm{mol}^{-1}$ ): calculated C 60.50, H 5.68, N 11.65\%; found C $60.66, \mathrm{H} 5.78$, N $11.93 \%$.

\section{Refinement}

Crystal data, data collection and structure refinement details are summarized in Table 7 . The $\mathrm{C}$-bound $\mathrm{H}$ atoms were included in calculated positions and treated as riding on their parent $\mathrm{C}$ atom: $\mathrm{C}-\mathrm{H}=0.94-0.98 \AA$ with $U_{\text {iso }}(\mathrm{H})=1.5 U_{\text {eq }}(\mathrm{C}-$ methyl) and $1.2 U_{\text {eq }}(\mathrm{C})$ for other $\mathrm{H}$ atoms.

With the STOE IPDS I, a one-circle diffractometer, for the triclinic system often only $93 \%$ of the Ewald sphere is accessible. Hence, for compound II the _diffrn_reflns_Laue_measured_fraction_full of 0.939 is below the required minimum of 0.95. For II, a small number of low-angle reflections, either in the shadow of the beam-stop or with bad agreement, were omitted during the final cycles of refinement.

\section{Acknowledgements}

HSE is grateful to the University of Neuchâtel for their support over the years.

\section{Funding information}

Funding for this research was provided by: Swiss National Science Foundation and the University of Neuchâtel.

\section{References}

Addison, A. W., Rao, T. N., Reedijk, J., van Rijn, J. \& Verschoor, G. C. (1984). J. Chem. Soc. Dalton Trans. pp. 1349-1356.

Graf, M., Greaves, B. \& Stoeckli-Evans, H. (1993). Inorg. Chim. Acta, 204, 239-246.

Graf, M. \& Stoeckli-Evans, H. (1994). Acta Cryst. C50, 1461-1464.

Groom, C. R., Bruno, I. J., Lightfoot, M. P. \& Ward, S. C. (2016). Acta Cryst. B72, 171-179.

Hong, X.-J., Feng, H.-X., Wei, M.-J., Peng, H.-J., Xie, J.-Q., Cai, Y.-P. \& Si, L.-P. (2017). Inorg. Chem. Commun. 77, 59-63.

Macrae, C. F., Sovago, I., Cottrell, S. J., Galek, P. T. A., McCabe, P., Pidcock, E., Platings, M., Shields, G. P., Stevens, J. S., Towler, M. \& Wood, P. A. (2020). J. Appl. Cryst. 53, 226-235.

McKinnon, J. J., Jayatilaka, D. \& Spackman, M. A. (2007). Chem. Commun. pp. 3814-3816.

Saghatforoush, L. (2015). Jiegou Hиахие, 34, 1869-1875.

Saljooghi, A. Sh. \& Fatemi, S. J. A. (2011). Russ. J. Coord. Chem. 37, 168-171. 
Sheldrick, G. M. (2008). Acta Cryst. A64, 112-122.

Sheldrick, G. M. (2015). Acta Cryst. C71, 3-8.

Spackman, M. A. \& Jayatilaka, D. (2009). CrystEngComm, 11, 1932.

Spek, A. L. (2020). Acta Cryst. E76, 1-11.

Stoe \& Cie (2004). IPDS-I Bedienungshandbuch. Stoe \& Cie GmbH, Darmstadt, Germany.

Tesouro Vallina, A. (2001). PhD Thesis. University of Neuchâtel, Switzerland.
Tesouro Vallina, A. \& Stoeckli-Evans, H. (2020). Acta Cryst. E76 $404-$ 409.

Trivedi, M., Pandey, D. S. \& Rath, N. P. (2009). Inorg. Chim. Acta, 362, 284-290.

Turner, M. J., McKinnon, J. J., Wolff, S. K., Grimwood, D. J., Spackman, P. R., Jayatilaka, D. \& Spackman, M. A. (2017). CrystalExplorer17. University of Western Australia. http://hirshfeldsurface.net

Westrip, S. P. (2010). J. Appl. Cryst. 43, 920-925. 


\section{supporting information}

Acta Cryst. (2020). E76, 410-416 [https://doi.org/10.1107/S2056989020001644]

The crystal structures and Hirshfeld surface analyses of a cadmium(II) and a zinc(II) mononuclear complex of the new tetrakis-substituted pyrazine ligand $N, N^{\prime}, N^{\prime \prime}, N^{\prime \prime \prime}$-[pyrazine-2,3,5,6-tetrayltetrakis(methylene)] tetrakis( $N$-methylaniline)

\section{Ana Tesouro Vallina and Helen Stoeckli-Evans}

Computing details

For both structures, data collection: EXPOSE in IPDS-I (Stoe \& Cie, 2004); cell refinement: CELL in IPDS-I (Stoe \& Cie, 2004); data reduction: INTEGRATE in IPDS-I (Stoe \& Cie, 2004); program(s) used to solve structure: SHELXS97 (Sheldrick, 2008); program(s) used to refine structure: SHELXL2018/3 (Sheldrick, 2015); molecular graphics: Mercury (Macrae et al., 2008). Software used to prepare material for publication: SHELXL2018/3 (Sheldrick, 2015), PLATON (Spek, 2020) and publCIF (Westrip, 2010) for (I); SHELXL2018/3 (Sheldrick, 2015), PLATON (Spek, 2009) and publCIF (Westrip, 2010) for (II).

Diiodido\{ $N, N^{\prime}, N^{\prime \prime}, N^{\prime \prime \prime}$-[pyrazine-2,3,5,6-tetrayltetrakis(methylene)] tetrakis( $N$-methylaniline)$\left.\boldsymbol{\kappa}^{3} N^{2}, N^{1}, N^{6}\right\}$ cadmium(II) (I)

Crystal data

$\left[\mathrm{CdI}_{2}\left(\mathrm{C}_{36} \mathrm{H}_{40} \mathrm{~N}_{6}\right)\right]$

$M_{r}=922.94$

Monoclinic, $I 2 / a$ $a=12.8370(7) \AA$

$b=20.1241(14) \AA$

$c=15.2568(9) \AA$

$\beta=110.871(6)^{\circ}$

$V=3682.7$ (4) $\AA^{3}$

$Z=4$

Data collection

STOE IPDS 1 diffractometer

Radiation source: fine-focus sealed tube Plane graphite monochromator $\varphi$ rotation scans Absorption correction: multi-scan

(MULABS; Spek, 2009)

$T_{\min }=0.961, T_{\max }=1.000$
$F(000)=1808$

$D_{\mathrm{x}}=1.665 \mathrm{Mg} \mathrm{m}^{-3}$

Mo $K \alpha$ radiation, $\lambda=0.71073 \AA$

Cell parameters from 5000 reflections

$\theta=1.7-26.1^{\circ}$

$\mu=2.30 \mathrm{~mm}^{-1}$

$T=223 \mathrm{~K}$

Rod, green

$0.40 \times 0.10 \times 0.10 \mathrm{~mm}$

14308 measured reflections

3566 independent reflections

2549 reflections with $I>2 \sigma(I)$

$R_{\text {int }}=0.031$

$\theta_{\text {max }}=25.9^{\circ}, \theta_{\min }=2.0^{\circ}$

$h=-15 \rightarrow 15$

$k=-24 \rightarrow 24$

$l=-18 \rightarrow 18$ 


\section{Refinement}

Refinement on $F^{2}$

Least-squares matrix: full

$R\left[F^{2}>2 \sigma\left(F^{2}\right)\right]=0.029$

$w R\left(F^{2}\right)=0.072$

$S=0.95$

3566 reflections

207 parameters

0 restraints

Primary atom site location: structure-invariant direct methods
Secondary atom site location: difference Fourier map

Hydrogen site location: inferred from neighbouring sites

$\mathrm{H}$-atom parameters constrained

$w=1 /\left[\sigma^{2}\left(F_{0}^{2}\right)+(0.0409 P)^{2}\right]$

where $P=\left(F_{\mathrm{o}}{ }^{2}+2 F_{\mathrm{c}}{ }^{2}\right) / 3$

$(\Delta / \sigma)_{\max }=0.001$

$\Delta \rho_{\max }=1.15 \mathrm{e} \AA^{-3}$

$\Delta \rho_{\min }=-0.88$ e $\AA^{-3}$

Special details

Geometry. All esds (except the esd in the dihedral angle between two 1.s. planes) are estimated using the full covariance matrix. The cell esds are taken into account individually in the estimation of esds in distances, angles and torsion angles; correlations between esds in cell parameters are only used when they are defined by crystal symmetry. An approximate (isotropic) treatment of cell esds is used for estimating esds involving l.s. planes.

Fractional atomic coordinates and isotropic or equivalent isotropic displacement parameters $\left(\AA^{2}\right)$

\begin{tabular}{lllll}
\hline & $x$ & $y$ & $z$ & $U_{\text {iso }}^{*} / U_{\text {eq }}$ \\
\hline Cd1 & 0.750000 & $0.06345(2)$ & 0.000000 & $0.04883(11)$ \\
I1 & $0.55787(2)$ & $0.11927(2)$ & $-0.00131(2)$ & $0.07922(12)$ \\
N1 & 0.750000 & $-0.05058(17)$ & 0.000000 & $0.0399(8)$ \\
N2 & $0.6906(2)$ & $0.01854(14)$ & $-0.17053(17)$ & $0.0460(6)$ \\
N3 & $0.7067(3)$ & $-0.26232(15)$ & $-0.15731(19)$ & $0.0545(7)$ \\
N4 & 0.750000 & $-0.18660(18)$ & 0.000000 & $0.0423(8)$ \\
C1 & $0.7472(2)$ & $-0.08374(16)$ & $-0.0768(2)$ & $0.0410(7)$ \\
C2 & $0.7432(3)$ & $-0.15288(16)$ & $-0.0772(2)$ & $0.0417(7)$ \\
C3 & $0.7572(3)$ & $-0.04292(16)$ & $-0.1562(2)$ & $0.0476(8)$ \\
H3A & 0.835630 & -0.031459 & -0.142427 & $0.057^{*}$ \\
H3B & 0.731715 & -0.069302 & -0.213986 & $0.057^{*}$ \\
C4 & $0.7161(3)$ & $0.06601(18)$ & $-0.2319(2)$ & $0.0513(8)$ \\
C5 & $0.7873(3)$ & $0.0528(2)$ & $-0.2792(3)$ & $0.0655(10)$ \\
H5 & 0.820979 & 0.010810 & -0.273599 & $0.079^{*}$ \\
C6 & $0.8096(4)$ & $0.1012(2)$ & $-0.3351(3)$ & $0.0838(14)$ \\
H6 & 0.858370 & 0.091568 & -0.367010 & $0.101^{*}$ \\
C7 & $0.7617(4)$ & $0.1625(3)$ & $-0.3441(4)$ & $0.0881(14)$ \\
H7 & 0.777532 & 0.195179 & -0.381596 & $0.106^{*}$ \\
C8 & $0.6897(4)$ & $0.1759(2)$ & $-0.2974(3)$ & $0.0835(13)$ \\
H8 & 0.655115 & 0.217760 & -0.304226 & $0.100^{*}$ \\
C9 & $0.6677(3)$ & $0.1284(2)$ & $-0.2406(3)$ & $0.0674(11)$ \\
H9 & 0.619914 & 0.138442 & -0.207941 & $0.081^{*}$ \\
C10 & $0.5702(3)$ & $0.0017(2)$ & $-0.2061(2)$ & $0.0591(9)$ \\
H10A & 0.550377 & -0.019190 & -0.267217 & $0.089^{*}$ \\
H10B & 0.526719 & 0.041903 & -0.211542 & $0.089^{*}$ \\
H10C & 0.554820 & -0.028697 & -0.162881 & $0.089^{*}$ \\
C11 & $0.7299(3)$ & $-0.19289(17)$ & $-0.1643(2)$ & $0.0528(8)$ \\
H11A & 0.669076 & -0.173622 & -0.217143 & $0.063^{*}$ \\
& & & & \\
\hline
\end{tabular}




$\begin{array}{lllll}\text { H11B } & 0.798405 & -0.188947 & -0.178391 & 0.063^{*} \\ \text { C12 } & 0.7924(3) & -0.30691(18) & -0.1149(2) & 0.0564(9) \\ \text { C13 } & 0.7708(5) & -0.3737(2) & -0.1026(3) & 0.0763(13) \\ \text { H13 } & 0.696946 & -0.389104 & -0.121410 & 0.092^{*} \\ \text { C14 } & 0.8600(6) & -0.4174(2) & -0.0619(4) & 0.0975(17) \\ \text { H14 } & 0.844606 & -0.462330 & -0.054507 & 0.117^{*} \\ \text { C15 } & 0.9680(6) & -0.3971(3) & -0.0330(4) & 0.1016(18) \\ \text { H15 } & 1.026535 & -0.427029 & -0.004708 & 0.122^{*} \\ \text { C16 } & 0.9892(4) & -0.3321(3) & -0.0459(3) & 0.0862^{*}(14) \\ \text { H16 } & 1.063444 & -0.317367 & -0.027305 & 0.103^{*} \\ \text { C17 } & 0.9041(4) & -0.2880(2) & -0.0856(3) & 0.0664(10) \\ \text { H17 } & 0.921527 & -0.243502 & -0.093253 & 0.080^{*} \\ \text { C18 } & 0.5935(4) & -0.2794(2) & -0.1693(3) & 0.0777(12) \\ \text { H18A } & 0.569896 & -0.317068 & -0.211386 & 0.117^{*} \\ \text { H18B } & 0.545221 & -0.241751 & -0.195689 & 0.117^{*} \\ \text { H18C } & 0.589042 & -0.290749 & -0.108965 & 0.117^{*}\end{array}$

Atomic displacement parameters $\left(\AA^{2}\right)$

\begin{tabular}{lllllll}
\hline & $U^{11}$ & $U^{22}$ & $U^{33}$ & $U^{12}$ & $U^{13}$ & $U^{23}$ \\
\hline Cd1 & $0.03359(17)$ & $0.0483(2)$ & $0.0628(2)$ & 0.000 & $0.01500(15)$ & 0.000 \\
I1 & $0.04735(16)$ & $0.0918(2)$ & $0.0946(2)$ & $0.02423(13)$ & $0.02050(14)$ & $-0.00291(16)$ \\
N1 & $0.0377(19)$ & $0.043(2)$ & $0.0401(19)$ & 0.000 & $0.0151(15)$ & 0.000 \\
N2 & $0.0346(13)$ & $0.0559(16)$ & $0.0469(14)$ & $0.0013(12)$ & $0.0136(11)$ & $0.0099(12)$ \\
N3 & $0.0613(18)$ & $0.0577(18)$ & $0.0493(16)$ & $-0.0119(15)$ & $0.0254(14)$ & $-0.0097(14)$ \\
N4 & $0.049(2)$ & $0.044(2)$ & $0.0371(19)$ & 0.000 & $0.0191(16)$ & 0.000 \\
C1 & $0.0357(15)$ & $0.0541(19)$ & $0.0358(15)$ & $0.0012(14)$ & $0.0157(12)$ & $0.0024(14)$ \\
C2 & $0.0398(16)$ & $0.0521(19)$ & $0.0373(16)$ & $0.0012(14)$ & $0.0187(13)$ & $-0.0003(14)$ \\
C3 & $0.0478(18)$ & $0.0541(19)$ & $0.0444(17)$ & $0.0018(15)$ & $0.0209(14)$ & $0.0078(14)$ \\
C4 & $0.0404(17)$ & $0.059(2)$ & $0.0513(18)$ & $-0.0002(15)$ & $0.0118(14)$ & $0.0126(16)$ \\
C5 & $0.057(2)$ & $0.076(3)$ & $0.071(2)$ & $0.0077(19)$ & $0.0325(19)$ & $0.024(2)$ \\
C6 & $0.074(3)$ & $0.098(3)$ & $0.093(3)$ & $0.013(3)$ & $0.046(3)$ & $0.040(3)$ \\
C7 & $0.075(3)$ & $0.094(3)$ & $0.099(3)$ & $0.003(3)$ & $0.036(3)$ & $0.045(3)$ \\
C8 & $0.071(3)$ & $0.070(3)$ & $0.104(3)$ & $0.010(2)$ & $0.025(3)$ & $0.033(3)$ \\
C9 & $0.052(2)$ & $0.068(2)$ & $0.081(3)$ & $0.0093(18)$ & $0.023(2)$ & $0.021(2)$ \\
C10 & $0.0371(18)$ & $0.077(3)$ & $0.058(2)$ & $-0.0061(17)$ & $0.0104(15)$ & $0.0104(18)$ \\
C11 & $0.068(2)$ & $0.056(2)$ & $0.0398(17)$ & $-0.0015(17)$ & $0.0263(16)$ & $-0.0037(15)$ \\
C12 & $0.080(3)$ & $0.055(2)$ & $0.0427(18)$ & $-0.0036(19)$ & $0.0322(18)$ & $-0.0075(15)$ \\
C13 & $0.113(4)$ & $0.060(3)$ & $0.064(2)$ & $-0.011(2)$ & $0.041(3)$ & $-0.009(2)$ \\
C14 & $0.161(6)$ & $0.059(3)$ & $0.084(3)$ & $0.018(3)$ & $0.058(4)$ & $0.005(2)$ \\
C15 & $0.126(5)$ & $0.101(5)$ & $0.088(4)$ & $0.042(4)$ & $0.051(4)$ & $0.004(3)$ \\
C16 & $0.084(3)$ & $0.107(4)$ & $0.074(3)$ & $0.021(3)$ & $0.035(2)$ & $-0.008(3)$ \\
C17 & $0.076(3)$ & $0.072(3)$ & $0.058(2)$ & $0.002(2)$ & $0.032(2)$ & $-0.0091(19)$ \\
C18 & $0.070(3)$ & $0.094(3)$ & $0.072(3)$ & $-0.023(2)$ & $0.029(2)$ & $-0.011(2)$ \\
& & & & & & \\
& & & & & &
\end{tabular}


Geometric parameters $\left(\AA,{ }^{\circ}\right)$

\begin{tabular}{|c|c|c|c|}
\hline $\mathrm{Cd} 1-\mathrm{N} 1$ & $2.295(3)$ & C6- 6 6 & 0.9400 \\
\hline $\mathrm{Cd} 1-\mathrm{N} 2^{\mathrm{i}}$ & $2.599(3)$ & $\mathrm{C} 7-\mathrm{C} 8$ & $1.380(7)$ \\
\hline $\mathrm{Cd} 1-\mathrm{N} 2$ & $2.599(3)$ & $\mathrm{C} 7-\mathrm{H} 7$ & 0.9400 \\
\hline $\mathrm{Cd} 1-\mathrm{I}^{\mathrm{i}}$ & $2.7038(3)$ & $\mathrm{C} 8-\mathrm{C} 9$ & $1.386(6)$ \\
\hline $\mathrm{Cd} 1-\mathrm{I} 1$ & $2.7038(3)$ & $\mathrm{C} 8-\mathrm{H} 8$ & 0.9400 \\
\hline $\mathrm{N} 1-\mathrm{C} 1$ & $1.338(3)$ & C9-H9 & 0.9400 \\
\hline $\mathrm{N} 1-\mathrm{Cl}^{\mathrm{i}}$ & $1.338(3)$ & $\mathrm{C} 10-\mathrm{H} 10 \mathrm{~A}$ & 0.9700 \\
\hline $\mathrm{N} 2-\mathrm{C} 4$ & $1.454(4)$ & $\mathrm{C} 10-\mathrm{H} 10 \mathrm{~B}$ & 0.9700 \\
\hline $\mathrm{N} 2-\mathrm{C} 3$ & $1.475(4)$ & $\mathrm{C} 10-\mathrm{H} 10 \mathrm{C}$ & 0.9700 \\
\hline $\mathrm{N} 2-\mathrm{C} 10$ & $1.484(4)$ & $\mathrm{C} 11-\mathrm{H} 11 \mathrm{~A}$ & 0.9800 \\
\hline N3-C12 & $1.388(5)$ & C11-H11B & 0.9800 \\
\hline N3-C11 & $1.440(4)$ & $\mathrm{C} 12-\mathrm{C} 17$ & $1.394(6)$ \\
\hline N3-C18 & $1.440(5)$ & $\mathrm{C} 12-\mathrm{C} 13$ & $1.398(5)$ \\
\hline $\mathrm{N} 4-\mathrm{C} 2$ & $1.335(4)$ & $\mathrm{C} 13-\mathrm{C} 14$ & $1.402(7)$ \\
\hline $\mathrm{N} 4-\mathrm{C} 2^{\mathrm{i}}$ & $1.335(4)$ & $\mathrm{C} 13-\mathrm{H} 13$ & 0.9400 \\
\hline $\mathrm{C} 1-\mathrm{C} 2$ & $1.392(5)$ & $\mathrm{C} 14-\mathrm{C} 15$ & $1.360(9)$ \\
\hline $\mathrm{C} 1-\mathrm{C} 3$ & $1.506(4)$ & $\mathrm{C} 14-\mathrm{H} 14$ & 0.9400 \\
\hline $\mathrm{C} 2-\mathrm{C} 11$ & $1.511(4)$ & $\mathrm{C} 15-\mathrm{C} 16$ & $1.364(8)$ \\
\hline $\mathrm{C} 3-\mathrm{H} 3 \mathrm{~A}$ & 0.9800 & C15-H15 & 0.9400 \\
\hline C $3-\mathrm{H} 3 \mathrm{~B}$ & 0.9800 & $\mathrm{C} 16-\mathrm{C} 17$ & $1.370(6)$ \\
\hline $\mathrm{C} 4-\mathrm{C} 5$ & $1.376(5)$ & $\mathrm{C} 16-\mathrm{H} 16$ & 0.9400 \\
\hline $\mathrm{C} 4-\mathrm{C} 9$ & $1.386(5)$ & C17-H17 & 0.9400 \\
\hline $\mathrm{C} 5-\mathrm{C} 6$ & $1.390(5)$ & $\mathrm{C} 18-\mathrm{H} 18 \mathrm{~A}$ & 0.9700 \\
\hline $\mathrm{C} 5-\mathrm{H} 5$ & 0.9400 & C18-H18B & 0.9700 \\
\hline $\mathrm{C} 6-\mathrm{C} 7$ & $1.363(7)$ & $\mathrm{C} 18-\mathrm{H} 18 \mathrm{C}$ & 0.9700 \\
\hline $\mathrm{N} 1-\mathrm{Cd} 1-\mathrm{N} 2^{\mathrm{i}}$ & $69.65(6)$ & $\mathrm{C} 6-\mathrm{C} 7-\mathrm{H} 7$ & 120.5 \\
\hline $\mathrm{N} 1-\mathrm{Cd} 1-\mathrm{N} 2$ & $69.65(6)$ & $\mathrm{C} 8-\mathrm{C} 7-\mathrm{H} 7$ & 120.5 \\
\hline $\mathrm{N} 2-\mathrm{Cd} 1-\mathrm{N} 2$ & $139.31(12)$ & $\mathrm{C} 7-\mathrm{C} 8-\mathrm{C} 9$ & $120.7(4)$ \\
\hline $\mathrm{N} 1-\mathrm{Cd} 1-\mathrm{I} 1^{\mathrm{i}}$ & $114.551(10)$ & $\mathrm{C} 7-\mathrm{C} 8-\mathrm{H} 8$ & 119.6 \\
\hline $\mathrm{N} 2^{\mathrm{i}}-\mathrm{Cd} 1-\mathrm{I} 1^{\mathrm{i}}$ & $101.29(6)$ & $\mathrm{C} 9-\mathrm{C} 8-\mathrm{H} 8$ & 119.6 \\
\hline $\mathrm{N} 1-\mathrm{Cd} 1-\mathrm{I} 1$ & $114.551(10)$ & $\mathrm{C} 8-\mathrm{C} 9-\mathrm{C} 4$ & $120.1(4)$ \\
\hline $\mathrm{N} 2{ }^{\mathrm{i}}-\mathrm{Cd} 1-\mathrm{I} 1$ & $95.35(6)$ & $\mathrm{C} 8-\mathrm{C} 9-\mathrm{H} 9$ & 120.0 \\
\hline $\mathrm{N} 2-\mathrm{Cd} 1-\mathrm{I} 1^{\mathrm{i}}$ & $95.35(6)$ & $\mathrm{C} 4-\mathrm{C} 9-\mathrm{H} 9$ & 120.0 \\
\hline $\mathrm{N} 2-\mathrm{Cd} 1-\mathrm{I} 1$ & $101.29(6)$ & $\mathrm{N} 2-\mathrm{C} 10-\mathrm{H} 10 \mathrm{~A}$ & 109.5 \\
\hline $\mathrm{I} 1^{\mathrm{i}}-\mathrm{Cd} 1-\mathrm{I} 1$ & $130.90(2)$ & $\mathrm{N} 2-\mathrm{C} 10-\mathrm{H} 10 \mathrm{~B}$ & 109.5 \\
\hline $\mathrm{C} 1-\mathrm{N} 1-\mathrm{C}^{\mathrm{i}}$ & $120.2(4)$ & $\mathrm{H} 10 \mathrm{~A}-\mathrm{C} 10-\mathrm{H} 10 \mathrm{~B}$ & 109.5 \\
\hline $\mathrm{C} 1-\mathrm{N} 1-\mathrm{Cd} 1$ & $119.92(19)$ & $\mathrm{N} 2-\mathrm{C} 10-\mathrm{H} 10 \mathrm{C}$ & 109.5 \\
\hline $\mathrm{C} 1-\mathrm{i} 1-\mathrm{Cd} 1$ & $119.92(19)$ & $\mathrm{H} 10 \mathrm{~A}-\mathrm{C} 10-\mathrm{H} 10 \mathrm{C}$ & 109.5 \\
\hline $\mathrm{C} 4-\mathrm{N} 2-\mathrm{C} 3$ & $113.3(3)$ & $\mathrm{H} 10 \mathrm{~B}-\mathrm{C} 10-\mathrm{H} 10 \mathrm{C}$ & 109.5 \\
\hline $\mathrm{C} 4-\mathrm{N} 2-\mathrm{C} 10$ & $111.0(2)$ & $\mathrm{N} 3-\mathrm{C} 11-\mathrm{C} 2$ & $114.4(3)$ \\
\hline $\mathrm{C} 3-\mathrm{N} 2-\mathrm{C} 10$ & $109.6(3)$ & $\mathrm{N} 3-\mathrm{C} 11-\mathrm{H} 11 \mathrm{~A}$ & 108.7 \\
\hline $\mathrm{C} 4-\mathrm{N} 2-\mathrm{Cd} 1$ & $111.3(2)$ & $\mathrm{C} 2-\mathrm{C} 11-\mathrm{H} 11 \mathrm{~A}$ & 108.7 \\
\hline $\mathrm{C} 3-\mathrm{N} 2-\mathrm{Cd} 1$ & $101.28(17)$ & $\mathrm{N} 3-\mathrm{C} 11-\mathrm{H} 11 \mathrm{~B}$ & 108.7 \\
\hline $\mathrm{C} 10-\mathrm{N} 2-\mathrm{Cd} 1$ & $110.0(2)$ & $\mathrm{C} 2-\mathrm{C} 11-\mathrm{H} 11 \mathrm{~B}$ & 108.7 \\
\hline $\mathrm{C} 12-\mathrm{N} 3-\mathrm{C} 11$ & $120.8(3)$ & $\mathrm{H} 11 \mathrm{~A}-\mathrm{C} 11-\mathrm{H} 11 \mathrm{~B}$ & 107.6 \\
\hline
\end{tabular}




\begin{tabular}{|c|c|c|c|}
\hline $\mathrm{C} 12-\mathrm{N} 3-\mathrm{C} 18$ & $120.1(3)$ & $\mathrm{N} 3-\mathrm{C} 12-\mathrm{C} 17$ & $121.8(3)$ \\
\hline $\mathrm{C} 11-\mathrm{N} 3-\mathrm{C} 18$ & $116.6(3)$ & $\mathrm{N} 3-\mathrm{C} 12-\mathrm{C} 13$ & $121.4(4)$ \\
\hline $\mathrm{C} 2-\mathrm{N} 4-\mathrm{C} 2^{\mathrm{i}}$ & $118.9(4)$ & $\mathrm{C} 17-\mathrm{C} 12-\mathrm{C} 13$ & $116.8(4)$ \\
\hline $\mathrm{N} 1-\mathrm{C} 1-\mathrm{C} 2$ & $119.5(3)$ & $\mathrm{C} 12-\mathrm{C} 13-\mathrm{C} 14$ & $119.6(5)$ \\
\hline $\mathrm{N} 1-\mathrm{C} 1-\mathrm{C} 3$ & $116.7(3)$ & $\mathrm{C} 12-\mathrm{C} 13-\mathrm{H} 13$ & 120.2 \\
\hline $\mathrm{C} 2-\mathrm{C} 1-\mathrm{C} 3$ & $123.7(3)$ & $\mathrm{C} 14-\mathrm{C} 13-\mathrm{H} 13$ & 120.2 \\
\hline $\mathrm{N} 4-\mathrm{C} 2-\mathrm{C} 1$ & $120.9(3)$ & $\mathrm{C} 15-\mathrm{C} 14-\mathrm{C} 13$ & $122.1(5)$ \\
\hline $\mathrm{N} 4-\mathrm{C} 2-\mathrm{C} 11$ & $117.2(3)$ & $\mathrm{C} 15-\mathrm{C} 14-\mathrm{H} 14$ & 118.9 \\
\hline $\mathrm{C} 1-\mathrm{C} 2-\mathrm{C} 11$ & $121.9(3)$ & $\mathrm{C} 13-\mathrm{C} 14-\mathrm{H} 14$ & 118.9 \\
\hline $\mathrm{N} 2-\mathrm{C} 3-\mathrm{C} 1$ & $111.4(3)$ & $\mathrm{C} 14-\mathrm{C} 15-\mathrm{C} 16$ & $118.4(5)$ \\
\hline $\mathrm{N} 2-\mathrm{C} 3-\mathrm{H} 3 \mathrm{~A}$ & 109.3 & $\mathrm{C} 14-\mathrm{C} 15-\mathrm{H} 15$ & 120.8 \\
\hline $\mathrm{C} 1-\mathrm{C} 3-\mathrm{H} 3 \mathrm{~A}$ & 109.3 & $\mathrm{C} 16-\mathrm{C} 15-\mathrm{H} 15$ & 120.8 \\
\hline $\mathrm{N} 2-\mathrm{C} 3-\mathrm{H} 3 \mathrm{~B}$ & 109.3 & $\mathrm{C} 15-\mathrm{C} 16-\mathrm{C} 17$ & $121.0(5)$ \\
\hline $\mathrm{C} 1-\mathrm{C} 3-\mathrm{H} 3 \mathrm{~B}$ & 109.3 & $\mathrm{C} 15-\mathrm{C} 16-\mathrm{H} 16$ & 119.5 \\
\hline $\mathrm{H} 3 \mathrm{~A}-\mathrm{C} 3-\mathrm{H} 3 \mathrm{~B}$ & 108.0 & $\mathrm{C} 17-\mathrm{C} 16-\mathrm{H} 16$ & 119.5 \\
\hline $\mathrm{C} 5-\mathrm{C} 4-\mathrm{C} 9$ & $118.9(3)$ & $\mathrm{C} 16-\mathrm{C} 17-\mathrm{C} 12$ & $122.1(4)$ \\
\hline $\mathrm{C} 5-\mathrm{C} 4-\mathrm{N} 2$ & $123.7(3)$ & $\mathrm{C} 16-\mathrm{C} 17-\mathrm{H} 17$ & 118.9 \\
\hline $\mathrm{C} 9-\mathrm{C} 4-\mathrm{N} 2$ & $117.4(3)$ & $\mathrm{C} 12-\mathrm{C} 17-\mathrm{H} 17$ & 118.9 \\
\hline $\mathrm{C} 4-\mathrm{C} 5-\mathrm{C} 6$ & $120.5(4)$ & $\mathrm{N} 3-\mathrm{C} 18-\mathrm{H} 18 \mathrm{~A}$ & 109.5 \\
\hline $\mathrm{C} 4-\mathrm{C} 5-\mathrm{H} 5$ & 119.8 & $\mathrm{~N} 3-\mathrm{C} 18-\mathrm{H} 18 \mathrm{~B}$ & 109.5 \\
\hline $\mathrm{C} 6-\mathrm{C} 5-\mathrm{H} 5$ & 119.8 & $\mathrm{H} 18 \mathrm{~A}-\mathrm{C} 18-\mathrm{H} 18 \mathrm{~B}$ & 109.5 \\
\hline $\mathrm{C} 7-\mathrm{C} 6-\mathrm{C} 5$ & $120.7(4)$ & $\mathrm{N} 3-\mathrm{C} 18-\mathrm{H} 18 \mathrm{C}$ & 109.5 \\
\hline $\mathrm{C} 7-\mathrm{C} 6-\mathrm{H} 6$ & 119.6 & $\mathrm{H} 18 \mathrm{~A}-\mathrm{C} 18-\mathrm{H} 18 \mathrm{C}$ & 109.5 \\
\hline $\mathrm{C} 5-\mathrm{C} 6-\mathrm{H} 6$ & 119.6 & $\mathrm{H} 18 \mathrm{~B}-\mathrm{C} 18-\mathrm{H} 18 \mathrm{C}$ & 109.5 \\
\hline $\mathrm{C} 6-\mathrm{C} 7-\mathrm{C} 8$ & $119.1(4)$ & & \\
\hline $\mathrm{C}{ }^{\mathrm{i}}-\mathrm{N} 1-\mathrm{C} 1-\mathrm{C} 2$ & $2.3(2)$ & $\mathrm{C} 4-\mathrm{C} 5-\mathrm{C} 6-\mathrm{C} 7$ & $0.0(7)$ \\
\hline $\mathrm{Cd} 1-\mathrm{N} 1-\mathrm{C} 1-\mathrm{C} 2$ & $-177.7(2)$ & $\mathrm{C} 5-\mathrm{C} 6-\mathrm{C} 7-\mathrm{C} 8$ & $-0.5(8)$ \\
\hline $\mathrm{C} 1 \mathrm{i}-\mathrm{N} 1-\mathrm{C} 1-\mathrm{C} 3$ & $-173.3(3)$ & $\mathrm{C} 6-\mathrm{C} 7-\mathrm{C} 8-\mathrm{C} 9$ & $1.2(7)$ \\
\hline $\mathrm{Cd} 1-\mathrm{N} 1-\mathrm{C} 1-\mathrm{C} 3$ & $6.7(3)$ & $\mathrm{C} 7-\mathrm{C} 8-\mathrm{C} 9-\mathrm{C} 4$ & $-1.6(7)$ \\
\hline $\mathrm{C} 2-\mathrm{i} 4-\mathrm{C} 2-\mathrm{C} 1$ & $2.3(2)$ & $\mathrm{C} 5-\mathrm{C} 4-\mathrm{C} 9-\mathrm{C} 8$ & $1.1(6)$ \\
\hline $\mathrm{C} 2{ }^{\mathrm{i}}-\mathrm{N} 4-\mathrm{C} 2-\mathrm{C} 11$ & $-177.0(3)$ & $\mathrm{N} 2-\mathrm{C} 4-\mathrm{C} 9-\mathrm{C} 8$ & $179.4(4)$ \\
\hline $\mathrm{N} 1-\mathrm{C} 1-\mathrm{C} 2-\mathrm{N} 4$ & $-4.7(4)$ & $\mathrm{C} 12-\mathrm{N} 3-\mathrm{C} 11-\mathrm{C} 2$ & $-82.7(4)$ \\
\hline $\mathrm{C} 3-\mathrm{C} 1-\mathrm{C} 2-\mathrm{N} 4$ & $170.6(3)$ & $\mathrm{C} 18-\mathrm{N} 3-\mathrm{C} 11-\mathrm{C} 2$ & $79.2(4)$ \\
\hline $\mathrm{N} 1-\mathrm{C} 1-\mathrm{C} 2-\mathrm{C} 11$ & $174.6(3)$ & $\mathrm{N} 4-\mathrm{C} 2-\mathrm{C} 11-\mathrm{N} 3$ & $10.3(4)$ \\
\hline $\mathrm{C} 3-\mathrm{C} 1-\mathrm{C} 2-\mathrm{C} 11$ & $-10.1(5)$ & $\mathrm{C} 1-\mathrm{C} 2-\mathrm{C} 11-\mathrm{N} 3$ & $-169.0(3)$ \\
\hline $\mathrm{C} 4-\mathrm{N} 2-\mathrm{C} 3-\mathrm{C} 1$ & $167.5(3)$ & $\mathrm{C} 11-\mathrm{N} 3-\mathrm{C} 12-\mathrm{C} 17$ & $-6.2(5)$ \\
\hline $\mathrm{C} 10-\mathrm{N} 2-\mathrm{C} 3-\mathrm{C} 1$ & $-68.0(3)$ & $\mathrm{C} 18-\mathrm{N} 3-\mathrm{C} 12-\mathrm{C} 17$ & $-167.4(3)$ \\
\hline $\mathrm{Cd} 1-\mathrm{N} 2-\mathrm{C} 3-\mathrm{C} 1$ & $48.2(3)$ & $\mathrm{C} 11-\mathrm{N} 3-\mathrm{C} 12-\mathrm{C} 13$ & $176.2(3)$ \\
\hline $\mathrm{N} 1-\mathrm{C} 1-\mathrm{C} 3-\mathrm{N} 2$ & $-41.9(4)$ & $\mathrm{C} 18-\mathrm{N} 3-\mathrm{C} 12-\mathrm{C} 13$ & $15.0(5)$ \\
\hline $\mathrm{C} 2-\mathrm{C} 1-\mathrm{C} 3-\mathrm{N} 2$ & $142.8(3)$ & $\mathrm{N} 3-\mathrm{C} 12-\mathrm{C} 13-\mathrm{C} 14$ & $178.1(4)$ \\
\hline $\mathrm{C} 3-\mathrm{N} 2-\mathrm{C} 4-\mathrm{C} 5$ & $5.8(4)$ & $\mathrm{C} 17-\mathrm{C} 12-\mathrm{C} 13-\mathrm{C} 14$ & $0.3(6)$ \\
\hline $\mathrm{C} 10-\mathrm{N} 2-\mathrm{C} 4-\mathrm{C} 5$ & $-118.0(4)$ & $\mathrm{C} 12-\mathrm{C} 13-\mathrm{C} 14-\mathrm{C} 15$ & $0.6(7)$ \\
\hline $\mathrm{Cd} 1-\mathrm{N} 2-\mathrm{C} 4-\mathrm{C} 5$ & $119.1(3)$ & $\mathrm{C} 13-\mathrm{C} 14-\mathrm{C} 15-\mathrm{C} 16$ & $-1.3(8)$ \\
\hline $\mathrm{C} 3-\mathrm{N} 2-\mathrm{C} 4-\mathrm{C} 9$ & $-172.4(3)$ & $\mathrm{C} 14-\mathrm{C} 15-\mathrm{C} 16-\mathrm{C} 17$ & $1.2(8)$ \\
\hline $\mathrm{C} 10-\mathrm{N} 2-\mathrm{C} 4-\mathrm{C} 9$ & $63.8(4)$ & $\mathrm{C} 15-\mathrm{C} 16-\mathrm{C} 17-\mathrm{C} 12$ & $-0.3(7)$ \\
\hline $\mathrm{Cd} 1-\mathrm{N} 2-\mathrm{C} 4-\mathrm{C} 9$ & $-59.1(3)$ & $\mathrm{N} 3-\mathrm{C} 12-\mathrm{C} 17-\mathrm{C} 16$ & $-178.2(3)$ \\
\hline
\end{tabular}




\begin{tabular}{llll}
$\mathrm{C} 9-\mathrm{C} 4-\mathrm{C} 5-\mathrm{C} 6$ & $-0.3(6)$ & $\mathrm{C} 13-\mathrm{C} 12-\mathrm{C} 17-\mathrm{C} 16$ & $-0.5(5)$ \\
$\mathrm{N} 2-\mathrm{C} 4-\mathrm{C} 5-\mathrm{C} 6$ & $-178.5(4)$ & & \\
\hline
\end{tabular}

Symmetry code: (i) $-x+3 / 2, y,-z$.

Hydrogen-bond geometry $\left(A,{ }^{\circ}\right)$

$\mathrm{Cg} 3$ is the centroid of the pyrazine ring $\mathrm{N} 1 / \mathrm{N} 4 / \mathrm{C} 1 / \mathrm{C} 2 / \mathrm{C}^{\mathrm{i}} / \mathrm{C} 2^{\mathrm{i}}$ and $\mathrm{Cg} 5$ is the centroid of the $\mathrm{C} 12-\mathrm{C} 17$ ring.

\begin{tabular}{lllll}
\hline$D-\mathrm{H} \cdots A$ & $D-\mathrm{H}$ & $\mathrm{H} \cdots A$ & $D \cdots A$ & $D-\mathrm{H} \cdots A$ \\
\hline $\mathrm{C} 18-\mathrm{H} 18 C \cdots \mathrm{Cg} 5^{\mathrm{i}}$ & 0.97 & 2.95 & $3.896(5)$ & 165 \\
$\mathrm{C} 17-\mathrm{H} 17 \cdots \mathrm{I} 1^{\mathrm{ii}}$ & 0.94 & 3.09 & $3.907(4)$ & 147 \\
$\mathrm{Cd} 1-\mathrm{I} 1 \cdots \mathrm{C} g^{\mathrm{iii}}$ & $2.70(1)$ & $3.96(1)$ & $6.5131(12)$ & $155(1)$ \\
$\mathrm{Cd} 1-\mathrm{I} 1 \cdots \mathrm{Cg} 3^{\mathrm{iv}}$ & $2.70(1)$ & $3.96(1)$ & $6.5131(12)$ & $155(1)$ \\
\hline
\end{tabular}

Symmetry codes: (i) $-x+3 / 2, y,-z$; (ii) $x+1 / 2,-y, z$; (iii) $-x+1,-y,-z$; (iv) $x-1 / 2,-y, z$.

Dichorido\{ $N, N^{\prime}, N^{\prime \prime}, N^{\prime \prime \prime}$-[pyrazine-2,3,5,6-tetrayltetrakis (methylene)] tetrakis( $N$-methylaniline)$\left.\boldsymbol{\kappa}^{3} N^{2}, N^{1}, N^{6}\right\}$ zinc(II) dichloromethane 0.6-solvate (II)

\section{Crystal data}

$\left[\mathrm{ZnCl}_{2}\left(\mathrm{C}_{36} \mathrm{H}_{40} \mathrm{~N}_{6}\right)\right] \cdot 0.6 \mathrm{CH}_{2} \mathrm{Cl}_{2}$

$M_{r}=743.99$

Triclinic, $P \overline{1}$

$a=11.9196(8) \AA$

$b=12.1208(8) \AA$

$c=13.919(1) \AA$

$\alpha=98.222(8)^{\circ}$

$\beta=100.313(8)^{\circ}$

$\gamma=107.580(7)^{\circ}$

$V=1843.9(2) \AA^{3}$

\section{Data collection}

STOE IPDS 1

diffractometer

Radiation source: fine-focus sealed tube

Plane graphite monochromator

$\varphi$ rotation scans

Absorption correction: multi-scan

(MULABS; Spek, 2009)

$T_{\min }=0.983, T_{\max }=1.000$

\section{Refinement}

Refinement on $F^{2}$

Least-squares matrix: full

$R\left[F^{2}>2 \sigma\left(F^{2}\right)\right]=0.043$

$w R\left(F^{2}\right)=0.117$

$S=0.79$

6654 reflections

437 parameters

0 restraints

Primary atom site location: structure-invariant

direct methods
$Z=2$

$F(000)=774.4$

$D_{\mathrm{x}}=1.340 \mathrm{Mg} \mathrm{m}^{-3}$

Mo $K \alpha$ radiation, $\lambda=0.71073 \AA$

Cell parameters from 5000 reflections

$\theta=1.7-26.1^{\circ}$

$\mu=0.93 \mathrm{~mm}^{-1}$

$T=223 \mathrm{~K}$

Rod, yellow

$0.30 \times 0.10 \times 0.10 \mathrm{~mm}$

14512 measured reflections

6654 independent reflections

3490 reflections with $I>2 \sigma(I)$

$R_{\text {int }}=0.054$

$\theta_{\text {max }}=25.9^{\circ}, \theta_{\min }=2.1^{\circ}$

$h=-14 \rightarrow 13$

$k=-13 \rightarrow 14$

$l=-17 \rightarrow 17$

Secondary atom site location: difference Fourier map

Hydrogen site location: inferred from

neighbouring sites

$\mathrm{H}$-atom parameters constrained

$w=1 /\left[\sigma^{2}\left(F_{0}^{2}\right)+(0.0649 P)^{2}\right]$

where $P=\left(F_{\mathrm{o}}^{2}+2 F_{\mathrm{c}}{ }^{2}\right) / 3$

$(\Delta / \sigma)_{\max }=0.028$

$\Delta \rho_{\max }=0.75$ e $\AA^{-3}$

$\Delta \rho_{\min }=-0.35$ e $\AA^{-3}$ 


\section{Special details}

Geometry. All esds (except the esd in the dihedral angle between two 1.s. planes) are estimated using the full covariance matrix. The cell esds are taken into account individually in the estimation of esds in distances, angles and torsion angles; correlations between esds in cell parameters are only used when they are defined by crystal symmetry. An approximate (isotropic) treatment of cell esds is used for estimating esds involving l.s. planes.

Fractional atomic coordinates and isotropic or equivalent isotropic displacement parameters $\left(\AA^{2}\right)$

\begin{tabular}{|c|c|c|c|c|c|}
\hline & $x$ & $y$ & $z$ & $U_{\text {iso }} * / U_{\text {eq }}$ & Occ. $(<1)$ \\
\hline Zn1 & $0.35680(4)$ & $0.17960(5)$ & $0.48236(4)$ & $0.03370(16)$ & \\
\hline $\mathrm{Cl1}$ & $0.26266(9)$ & $0.31262(9)$ & $0.48265(8)$ & $0.0379(3)$ & \\
\hline $\mathrm{Cl} 2$ & $0.33732(10)$ & $0.02609(9)$ & $0.55847(8)$ & $0.0383(3)$ & \\
\hline N1 & $0.4877(3)$ & $0.2055(3)$ & $0.4016(2)$ & $0.0294(8)$ & \\
\hline $\mathrm{N} 2$ & $0.2552(3)$ & $0.0630(3)$ & $0.3191(2)$ & $0.0354(8)$ & \\
\hline N3 & $0.6536(3)$ & $0.0643(4)$ & $0.1349(3)$ & $0.0463(10)$ & \\
\hline N4 & $0.6673(3)$ & $0.2247(3)$ & $0.3005(2)$ & $0.0353(9)$ & \\
\hline N5 & $0.5392(3)$ & $0.3173(3)$ & $0.5955(2)$ & $0.0297(8)$ & \\
\hline N6 & $0.9012(3)$ & $0.3813(4)$ & $0.3867(3)$ & $0.0440(10)$ & \\
\hline $\mathrm{C} 1$ & 0.4709 (4) & $0.1232(4)$ & $0.3201(3)$ & $0.0309(10)$ & \\
\hline $\mathrm{C} 2$ & $0.5649(4)$ & $0.1337(4)$ & $0.2698(3)$ & $0.0318(10)$ & \\
\hline $\mathrm{C} 3$ & $0.3531(4)$ & $0.0225(4)$ & $0.2939(3)$ & $0.0370(10)$ & \\
\hline $\mathrm{H} 3 \mathrm{~A}$ & 0.332467 & -0.012157 & 0.222331 & $0.044 *$ & \\
\hline $\mathrm{H} 3 \mathrm{~B}$ & 0.361523 & -0.039048 & 0.330527 & $0.044^{*}$ & \\
\hline $\mathrm{C} 4$ & $0.1483(4)$ & -0.0309 (4) & $0.3234(3)$ & $0.0380(11)$ & \\
\hline $\mathrm{C} 5$ & $0.1318(4)$ & $-0.1506(4)$ & $0.2941(3)$ & $0.0440(11)$ & \\
\hline H5 & 0.189961 & -0.173802 & 0.267030 & $0.053^{*}$ & \\
\hline C6 & $0.0295(4)$ & $-0.2354(5)$ & $0.3049(3)$ & $0.0524(13)$ & \\
\hline H6 & 0.020101 & -0.315972 & 0.286843 & $0.063 *$ & \\
\hline $\mathrm{C} 7$ & $-0.0586(5)$ & $-0.2035(6)$ & $0.3417(4)$ & $0.0615(15)$ & \\
\hline $\mathrm{H} 7$ & -0.128003 & -0.261909 & 0.348052 & $0.074 *$ & \\
\hline $\mathrm{C} 8$ & $-0.0441(4)$ & $-0.0849(6)$ & $0.3691(4)$ & $0.0618(15)$ & \\
\hline $\mathrm{H} 8$ & -0.104135 & -0.062684 & 0.393942 & $0.074 *$ & \\
\hline C9 & $0.0583(4)$ & $0.0012(5)$ & $0.3603(4)$ & $0.0518(13)$ & \\
\hline H9 & 0.067382 & 0.081656 & 0.379134 & $0.062 *$ & \\
\hline $\mathrm{C} 10$ & $0.2243(4)$ & $0.1374(4)$ & $0.2491(3)$ & $0.0492(12)$ & \\
\hline $\mathrm{H} 10 \mathrm{C}$ & 0.195802 & 0.090689 & 0.181450 & $0.074 *$ & \\
\hline $\mathrm{H} 10 \mathrm{~B}$ & 0.295684 & 0.204609 & 0.253252 & $0.074 *$ & \\
\hline H10A & 0.161260 & 0.165493 & 0.267231 & $0.074 *$ & \\
\hline C11 & $0.5545(4)$ & $0.0370(4)$ & $0.1832(3)$ & $0.0458(12)$ & \\
\hline H11A & 0.549429 & -0.035832 & 0.207651 & $0.055^{*}$ & \\
\hline H11B & 0.479057 & 0.021761 & 0.133629 & $0.055^{*}$ & \\
\hline $\mathrm{C} 12$ & $0.6568(4)$ & $0.1362(4)$ & $0.0662(3)$ & $0.0447(12)$ & \\
\hline $\mathrm{C} 13$ & $0.7525(5)$ & $0.1612(5)$ & $0.0174(4)$ & $0.0666(16)$ & \\
\hline H13 & 0.814974 & 0.129703 & 0.032399 & $0.080^{*}$ & \\
\hline C14 & $0.7552(7)$ & $0.2312(7)$ & $-0.0519(5)$ & $0.087(2)$ & \\
\hline H14 & 0.820382 & 0.246888 & -0.083057 & $0.105^{*}$ & \\
\hline $\mathrm{C} 15$ & $0.6682(9)$ & $0.2781(6)$ & $-0.0769(5)$ & 0.098 & \\
\hline H15 & 0.671980 & 0.325867 & -0.124647 & $0.117^{*}$ & \\
\hline
\end{tabular}




\begin{tabular}{|c|c|c|c|c|}
\hline C16 & $0.5726(7)$ & $0.2540(6)$ & $-0.0303(4)$ & $0.086(2)$ \\
\hline H16 & 0.510787 & 0.285978 & -0.046768 & $0.103^{*}$ \\
\hline $\mathrm{C} 17$ & $0.5667(5)$ & $0.1833(5)$ & 0.0405 (4) & 0.0599 (14) \\
\hline $\mathrm{H} 17$ & 0.500786 & 0.167545 & 0.070873 & $0.072 *$ \\
\hline C18 & $0.7620(6)$ & $0.0419(6)$ & 0.1793 (4) & $0.086(2)$ \\
\hline $\mathrm{H} 18 \mathrm{C}$ & 0.779738 & -0.012043 & 0.130429 & $0.129 *$ \\
\hline H18B & 0.749440 & 0.006775 & 0.236560 & $0.129^{*}$ \\
\hline H18A & 0.829462 & 0.115809 & 0.200454 & $0.129 *$ \\
\hline $\mathrm{C} 21$ & $0.5910(4)$ & $0.2969(4)$ & $0.4341(3)$ & $0.0288(9)$ \\
\hline $\mathrm{C} 22$ & 0.6828 & $0.3080(4)$ & $0.3814(3)$ & $0.0310(10)$ \\
\hline $\mathrm{C} 23$ & $0.5972(4)$ & $0.3833(4)$ & $0.5261(3)$ & $0.0324(10)$ \\
\hline $\mathrm{H} 23 \mathrm{~A}$ & 0.681896 & 0.429429 & 0.558643 & $0.039^{*}$ \\
\hline $\mathrm{H} 23 \mathrm{~B}$ & 0.555559 & 0.438161 & 0.507247 & $0.039^{*}$ \\
\hline $\mathrm{C} 24$ & $0.5072(4)$ & $0.3885(4)$ & $0.6722(3)$ & $0.0317(10)$ \\
\hline $\mathrm{C} 25$ & $0.5468(4)$ & $0.5100(4)$ & 0.6919 (3) & $0.0362(10)$ \\
\hline $\mathrm{H} 25$ & 0.599552 & 0.551869 & 0.656583 & $0.043^{*}$ \\
\hline $\mathrm{C} 26$ & $0.5080(4)$ & $0.5711(4)$ & $0.7651(3)$ & $0.0448(12)$ \\
\hline $\mathrm{H} 26$ & 0.533850 & 0.654230 & 0.778053 & $0.054^{*}$ \\
\hline $\mathrm{C} 27$ & $0.4327(4)$ & $0.5106(5)$ & $0.8179(3)$ & $0.0487(13)$ \\
\hline $\mathrm{H} 27$ & 0.407923 & 0.552419 & 0.867503 & $0.058^{*}$ \\
\hline $\mathrm{C} 28$ & $0.3934(4)$ & $0.3893(5)$ & 0.7988 (3) & $0.0489(12)$ \\
\hline $\mathrm{H} 28$ & 0.341885 & 0.347757 & 0.835120 & $0.059^{*}$ \\
\hline $\mathrm{C} 29$ & $0.4302(4)$ & $0.3282(4)$ & $0.7255(3)$ & $0.0440(12)$ \\
\hline $\mathrm{H} 29$ & 0.402641 & 0.245046 & 0.711871 & $0.053^{*}$ \\
\hline $\mathrm{C} 30$ & 0.6175 & $0.2547(4)$ & $0.6402(3)$ & $0.0432(12)$ \\
\hline $\mathrm{H} 30 \mathrm{C}$ & 0.693683 & 0.312083 & 0.679612 & $0.065^{*}$ \\
\hline H30B & 0.632752 & 0.203586 & 0.587463 & $0.065^{*}$ \\
\hline $\mathrm{H} 30 \mathrm{~A}$ & 0.577342 & 0.207377 & 0.682678 & $0.065^{*}$ \\
\hline C31 & 0.7994 & $0.4100(4)$ & $0.4112(3)$ & $0.0463(12)$ \\
\hline $\mathrm{H} 31 \mathrm{~A}$ & 0.788908 & 0.473627 & 0.378260 & $0.056^{*}$ \\
\hline H31B & 0.817746 & 0.440047 & 0.483454 & $0.056^{*}$ \\
\hline $\mathrm{C} 32$ & $0.9419(4)$ & $0.4069(4)$ & $0.3029(3)$ & $0.0368(10)$ \\
\hline C33 & $1.0564(4)$ & $0.4040(5)$ & $0.2933(3)$ & $0.0509(13)$ \\
\hline H33 & 1.103887 & 0.380026 & 0.342028 & $0.061^{*}$ \\
\hline C34 & $1.0987(5)$ & $0.4367(6)$ & $0.2120(4)$ & $0.0689(17)$ \\
\hline H34 & 1.175749 & 0.434902 & 0.207024 & $0.083^{*}$ \\
\hline C35 & $1.0337(5)$ & $0.4715(5)$ & 0.1385 (4) & $0.0710(17)$ \\
\hline H35 & 1.065545 & 0.495398 & 0.084821 & $0.085^{*}$ \\
\hline $\mathrm{C} 36$ & $0.9177(5)$ & $0.4703(5)$ & 0.1459 (4) & $0.0608(14)$ \\
\hline H36 & 0.869357 & 0.490972 & 0.095273 & $0.073^{*}$ \\
\hline $\mathrm{C} 37$ & $0.8741(4)$ & 0.4395 (4) & $0.2261(3)$ & $0.0480(12)$ \\
\hline H37 & 0.796333 & 0.440162 & 0.229773 & $0.058^{*}$ \\
\hline C38 & $0.9460(5)$ & $0.3087(5)$ & $0.4473(4)$ & $0.0611(15)$ \\
\hline $\mathrm{H} 38 \mathrm{C}$ & 0.951629 & 0.241231 & 0.404214 & $0.092^{*}$ \\
\hline H38B & 0.890860 & 0.281192 & 0.489074 & $0.092^{*}$ \\
\hline H38A & 1.025495 & 0.355234 & 0.489222 & $0.092 *$ \\
\hline $\mathrm{C} 40$ & $0.1063(10)$ & $0.2516(11)$ & $0.9543(8)$ & 0.095 (4) \\
\hline $\mathrm{H} 40 \mathrm{~A}$ & 0.053445 & 0.294842 & 0.973082 & $0.114 *$ \\
\hline
\end{tabular}




\begin{tabular}{llllll}
$\mathrm{H} 40 \mathrm{~B}$ & 0.103192 & 0.249458 & 0.883154 & $0.114^{*}$ & 0.6 \\
$\mathrm{Cl} 3$ & $0.2564(2)$ & $0.3346(3)$ & $1.02194(16)$ & $0.0912(10)$ & 0.6 \\
$\mathrm{Cl} 4$ & $0.0491(3)$ & $0.1163(3)$ & $0.9673(2)$ & $0.1003(10)$ & 0.6 \\
\hline
\end{tabular}

Atomic displacement parameters $\left(\AA^{2}\right)$

\begin{tabular}{|c|c|c|c|c|c|c|}
\hline & $U^{11}$ & $U^{22}$ & $U^{33}$ & $U^{12}$ & $U^{13}$ & $U^{23}$ \\
\hline $\mathrm{Zn} 1$ & $0.0365(3)$ & 0.0345 (3) & $0.0385(3)$ & $0.0163(3)$ & $0.0187(2)$ & $0.0121(2)$ \\
\hline $\mathrm{Cl1}$ & $0.0334(6)$ & $0.0367(7)$ & $0.0497(6)$ & $0.0193(6)$ & $0.0121(5)$ & $0.0092(5)$ \\
\hline $\mathrm{Cl} 2$ & $0.0485(7)$ & $0.0319(6)$ & $0.0394(6)$ & $0.0134(6)$ & $0.0156(5)$ & $0.0167(5)$ \\
\hline N1 & $0.032(2)$ & $0.031(2)$ & $0.0280(18)$ & $0.0121(19)$ & $0.0103(15)$ & $0.0056(15)$ \\
\hline $\mathrm{N} 2$ & $0.032(2)$ & $0.033(2)$ & $0.044(2)$ & $0.0111(19)$ & $0.0145(16)$ & $0.0112(16)$ \\
\hline N3 & $0.052(2)$ & 0.052 & $0.043(2)$ & $0.023(2)$ & 0.0239 (19) & $0.0053(19)$ \\
\hline N4 & $0.037(2)$ & $0.035(2)$ & $0.036(2)$ & $0.011(2)$ & $0.0168(16)$ & $0.0051(17)$ \\
\hline N5 & $0.037(2)$ & $0.029(2)$ & $0.0307(18)$ & $0.0164(18)$ & $0.0140(15)$ & $0.0092(15)$ \\
\hline N6 & $0.034(2)$ & 0.059 & $0.046(2)$ & $0.018(2)$ & $0.0189(18)$ & $0.0173(19)$ \\
\hline $\mathrm{C} 1$ & $0.034(2)$ & 0.028 & $0.033(2)$ & $0.011(2)$ & $0.0123(18)$ & $0.0089(19)$ \\
\hline $\mathrm{C} 2$ & $0.037(3)$ & $0.029(3)$ & $0.033(2)$ & $0.013(2)$ & $0.0150(19)$ & $0.0061(18)$ \\
\hline $\mathrm{C} 3$ & $0.037(3)$ & $0.031(3)$ & $0.042(3)$ & $0.010(2)$ & $0.013(2)$ & $0.003(2)$ \\
\hline $\mathrm{C} 4$ & $0.032(2)$ & $0.038(3)$ & $0.040(2)$ & $0.005(2)$ & 0.0093 (19) & $0.010(2)$ \\
\hline $\mathrm{C} 5$ & $0.044(3)$ & $0.037(3)$ & $0.046(3)$ & $0.007(3)$ & $0.011(2)$ & 0.007 (2) \\
\hline C6 & $0.050(3)$ & 0.043 & 0.050 & $-0.002(3)$ & $0.006(2)$ & 0.009 (2) \\
\hline $\mathrm{C} 7$ & $0.042(3)$ & $0.071(5)$ & 0.055 & -0.007 (3) & 0.013 & $0.015(3)$ \\
\hline $\mathrm{C} 8$ & $0.036(3)$ & $0.079(5)$ & 0.063 & 0.007 (3) & $0.020(2)$ & $0.006(3)$ \\
\hline C9 & $0.038(3)$ & 0.051 & $0.064(3)$ & 0.011 & $0.019(2)$ & $0.003(3)$ \\
\hline $\mathrm{C} 10$ & $0.049(3)$ & 0.048 & 0.049 (3) & 0.015 & $0.005(2)$ & $0.019(2)$ \\
\hline C11 & $0.055(3)$ & 0.038 & $0.044(3)$ & $0.012(3)$ & $0.024(2)$ & -0.001 \\
\hline $\mathrm{C} 12$ & $0.052(3)$ & $0.046(3)$ & $0.029(2)$ & 0.011 & $0.012(2)$ & $-0.006(2)$ \\
\hline $\mathrm{C} 13$ & $0.060(3)$ & $0.078(5)$ & $0.044(3)$ & $-0.003(3)$ & $0.024(3)$ & -0.002 \\
\hline C14 & $0.090(5)$ & $0.095(6)$ & $0.047(4)$ & $-0.012(5)$ & $0.026(4)$ & $0.002(4)$ \\
\hline $\mathrm{C} 15$ & $0.149(8)$ & $0.071(5)$ & $0.036(4)$ & $-0.011(5)$ & $0.017(4)$ & $0.005(3)$ \\
\hline $\mathrm{C} 16$ & $0.141(7)$ & $0.066(4)$ & $0.038(3)$ & $0.042(5)$ & $-0.004(4)$ & -0.007 \\
\hline $\mathrm{C} 17$ & $0.080(4)$ & $0.058(4)$ & $0.039(3)$ & 0.025 & $0.014(3)$ & -0.001 \\
\hline $\mathrm{C} 18$ & $0.086(4)$ & $0.122(6)$ & $0.081(4)$ & $0.072(5)$ & $0.032(4)$ & $0.023(4)$ \\
\hline $\mathrm{C} 21$ & $0.032(2)$ & $0.028(3)$ & $0.028(2)$ & $0.011(2)$ & $0.0081(18)$ & $0.0060(18)$ \\
\hline $\mathrm{C} 22$ & $0.028(2)$ & $0.032(3)$ & $0.036(2)$ & $0.011(2)$ & $0.0136(18)$ & $0.0078(19)$ \\
\hline $\mathrm{C} 23$ & $0.033(2)$ & $0.031(3)$ & $0.035(2)$ & $0.011(2)$ & $0.0126(19)$ & $0.0058(19)$ \\
\hline $\mathrm{C} 24$ & $0.033(2)$ & 0.035 & $0.028(2)$ & $0.015(2)$ & $0.0075(18)$ & $0.0027(18)$ \\
\hline $\mathrm{C} 25$ & $0.039(3)$ & 0.038 & $0.034(2)$ & $0.016(2)$ & 0.0104 (19) & $0.006(2)$ \\
\hline $\mathrm{C} 26$ & $0.056(3)$ & $0.042(3)$ & $0.038(3)$ & $0.020(3)$ & $0.014(2)$ & -0.001 \\
\hline $\mathrm{C} 27$ & $0.054(3)$ & $0.060(4)$ & $0.036(3)$ & $0.029(3)$ & $0.014(2)$ & -0.002 \\
\hline $\mathrm{C} 28$ & $0.053(3)$ & $0.060(4)$ & $0.040(3)$ & $0.021(3)$ & $0.023(2)$ & $0.010(2)$ \\
\hline C29 & $0.058(3)$ & 0.040 & $0.038(3)$ & $0.016(3)$ & $0.023(2)$ & $0.006(2)$ \\
\hline $\mathrm{C} 30$ & $0.050(3)$ & 0.050 & $0.039(3)$ & $0.031(3)$ & $0.009(2)$ & $0.008(2)$ \\
\hline C31 & 0.039 & $0.046(3)$ & $0.052(3)$ & 0.008 (3) & $0.025(2)$ & $-0.001(2)$ \\
\hline $\mathrm{C} 32$ & $0.031(2)$ & 0.038 & $0.036(2)$ & $0.007(2)$ & 0.0075 (19) & $-0.001(2)$ \\
\hline $\mathrm{C} 33$ & 0.039 & $0.076(4)$ & $0.040(3)$ & $0.021(3)$ & $0.015(2)$ & $0.008(2)$ \\
\hline C34 & $0.048(3)$ & $0.105(5)$ & $0.052(3)$ & $0.021(4)$ & $0.024(3)$ & 0.007 (3) \\
\hline
\end{tabular}




\begin{tabular}{lllllll}
\hline C35 & $0.076(4)$ & $0.092(5)$ & $0.041(3)$ & $0.016(4)$ & $0.027(3)$ & $0.011(3)$ \\
C36 & $0.077(4)$ & $0.051(4)$ & $0.046(3)$ & $0.012(3)$ & $0.008(3)$ & $0.015(3)$ \\
C37 & $0.043(3)$ & $0.045(3)$ & $0.054(3)$ & $0.012(3)$ & $0.011(2)$ & $0.011(2)$ \\
C38 & $0.046(3)$ & $0.082(4)$ & $0.058(3)$ & $0.019(3)$ & $0.013(3)$ & $0.030(3)$ \\
C40 & $0.082(8)$ & $0.114(10)$ & $0.084(8)$ & $0.037(8)$ & $-0.013(6)$ & $0.038(7)$ \\
C13 & $0.0444(13)$ & $0.179(3)$ & $0.0384(12)$ & $0.0251(17)$ & $0.0150(10)$ & $0.0076(15)$ \\
C14 & $0.088(2)$ & $0.098(3)$ & $0.115(2)$ & $0.043(2)$ & $0.0134(18)$ & $0.0124(19)$ \\
\hline
\end{tabular}

Geometric parameters $\left(\AA,{ }^{\circ}\right)$

\begin{tabular}{|c|c|c|c|}
\hline $\mathrm{Zn} 1-\mathrm{N} 1$ & $2.057(3)$ & $\mathrm{C} 15-\mathrm{C} 16$ & $1.386(10)$ \\
\hline $\mathrm{Zn} 1-\mathrm{N} 2$ & $2.385(3)$ & $\mathrm{C} 15-\mathrm{H} 15$ & 0.9400 \\
\hline $\mathrm{Zn} 1-\mathrm{N} 5$ & $2.413(3)$ & $\mathrm{C} 16-\mathrm{C} 17$ & $1.394(8)$ \\
\hline $\mathrm{Zn} 1-\mathrm{Cl1}$ & $2.2251(10)$ & $\mathrm{C} 16-\mathrm{H} 16$ & 0.9400 \\
\hline $\mathrm{Zn} 1-\mathrm{Cl} 2$ & $2.2425(11)$ & C17-H17 & 0.9400 \\
\hline $\mathrm{N} 1-\mathrm{C} 21$ & $1.334(5)$ & $\mathrm{C} 18-\mathrm{H} 18 \mathrm{C}$ & 0.9700 \\
\hline $\mathrm{N} 1-\mathrm{C} 1$ & $1.340(5)$ & $\mathrm{C} 18-\mathrm{H} 18 \mathrm{~B}$ & 0.9700 \\
\hline $\mathrm{N} 2-\mathrm{C} 4$ & $1.446(5)$ & $\mathrm{C} 18-\mathrm{H} 18 \mathrm{~A}$ & 0.9700 \\
\hline $\mathrm{N} 2-\mathrm{C} 3$ & $1.473(5)$ & $\mathrm{C} 21-\mathrm{C} 22$ & $1.407(5)$ \\
\hline $\mathrm{N} 2-\mathrm{C} 10$ & $1.491(5)$ & $\mathrm{C} 21-\mathrm{C} 23$ & $1.509(5)$ \\
\hline $\mathrm{N} 3-\mathrm{C} 12$ & $1.381(6)$ & $\mathrm{C} 22-\mathrm{C} 31$ & $1.497(6)$ \\
\hline $\mathrm{N} 3-\mathrm{C} 18$ & $1.449(6)$ & $\mathrm{C} 23-\mathrm{H} 23 \mathrm{~A}$ & 0.9800 \\
\hline $\mathrm{N} 3-\mathrm{C} 11$ & $1.438(5)$ & $\mathrm{C} 23-\mathrm{H} 23 \mathrm{~B}$ & 0.9800 \\
\hline $\mathrm{N} 4-\mathrm{C} 2$ & $1.325(5)$ & $\mathrm{C} 24-\mathrm{C} 25$ & $1.373(6)$ \\
\hline $\mathrm{N} 4-\mathrm{C} 22$ & $1.345(5)$ & $\mathrm{C} 24-\mathrm{C} 29$ & $1.386(6)$ \\
\hline $\mathrm{N} 5-\mathrm{C} 24$ & $1.453(5)$ & $\mathrm{C} 25-\mathrm{C} 26$ & $1.399(5)$ \\
\hline $\mathrm{N} 5-\mathrm{C} 30$ & $1.480(5)$ & $\mathrm{C} 25-\mathrm{H} 25$ & 0.9400 \\
\hline $\mathrm{N} 5-\mathrm{C} 23$ & $1.476(5)$ & $\mathrm{C} 26-\mathrm{C} 27$ & $1.371(7)$ \\
\hline $\mathrm{N} 6-\mathrm{C} 32$ & $1.380(5)$ & $\mathrm{C} 26-\mathrm{H} 26$ & 0.9400 \\
\hline N6-C31 & $1.441(5)$ & $\mathrm{C} 27-\mathrm{C} 28$ & $1.371(7)$ \\
\hline $\mathrm{N} 6-\mathrm{C} 38$ & $1.451(6)$ & $\mathrm{C} 27-\mathrm{H} 27$ & 0.9400 \\
\hline $\mathrm{C} 1-\mathrm{C} 2$ & $1.408(5)$ & $\mathrm{C} 28-\mathrm{C} 29$ & $1.385(6)$ \\
\hline $\mathrm{C} 1-\mathrm{C} 3$ & $1.500(6)$ & $\mathrm{C} 28-\mathrm{H} 28$ & 0.9400 \\
\hline $\mathrm{C} 2-\mathrm{C} 11$ & $1.516(5)$ & $\mathrm{C} 29-\mathrm{H} 29$ & 0.9400 \\
\hline $\mathrm{C} 3-\mathrm{H} 3 \mathrm{~A}$ & 0.9800 & $\mathrm{C} 30-\mathrm{H} 30 \mathrm{C}$ & 0.9700 \\
\hline С $3-\mathrm{H} 3 \mathrm{~B}$ & 0.9800 & С $30-\mathrm{H} 30 \mathrm{~B}$ & 0.9700 \\
\hline $\mathrm{C} 4-\mathrm{C} 5$ & $1.393(6)$ & $\mathrm{C} 30-\mathrm{H} 30 \mathrm{~A}$ & 0.9700 \\
\hline $\mathrm{C} 4-\mathrm{C} 9$ & $1.400(6)$ & $\mathrm{C} 31-\mathrm{H} 31 \mathrm{~A}$ & 0.9800 \\
\hline $\mathrm{C} 5-\mathrm{C} 6$ & $1.385(6)$ & С $31-\mathrm{H} 31 \mathrm{~B}$ & 0.9800 \\
\hline $\mathrm{C} 5-\mathrm{H} 5$ & 0.9400 & $\mathrm{C} 32-\mathrm{C} 37$ & $1.399(6)$ \\
\hline $\mathrm{C} 6-\mathrm{C} 7$ & $1.376(7)$ & $\mathrm{C} 32-\mathrm{C} 33$ & $1.404(6)$ \\
\hline C6- 6 6 & 0.9400 & $\mathrm{C} 33-\mathrm{C} 34$ & $1.380(7)$ \\
\hline $\mathrm{C} 7-\mathrm{C} 8$ & $1.383(8)$ & C $33-\mathrm{H} 33$ & 0.9400 \\
\hline $\mathrm{C} 7-\mathrm{H} 7$ & 0.9400 & $\mathrm{C} 34-\mathrm{C} 35$ & $1.365(7)$ \\
\hline $\mathrm{C} 8-\mathrm{C} 9$ & $1.384(7)$ & C34-H34 & 0.9400 \\
\hline $\mathrm{C} 8-\mathrm{H} 8$ & 0.9400 & $\mathrm{C} 35-\mathrm{C} 36$ & $1.401(7)$ \\
\hline C9- $\mathrm{H} 9$ & 0.9400 & C35-H35 & 0.9400 \\
\hline $\mathrm{C} 10-\mathrm{H} 10 \mathrm{C}$ & 0.9700 & $\mathrm{C} 36-\mathrm{C} 37$ & $1.365(7)$ \\
\hline
\end{tabular}




\begin{tabular}{|c|c|c|c|}
\hline $\mathrm{C} 10-\mathrm{H} 10 \mathrm{~B}$ & 0.9700 & $\mathrm{C} 36-\mathrm{H} 36$ & 0.9400 \\
\hline $\mathrm{C} 10-\mathrm{H} 10 \mathrm{~A}$ & 0.9700 & C $37-\mathrm{H} 37$ & 0.9400 \\
\hline $\mathrm{C} 11-\mathrm{H} 11 \mathrm{~A}$ & 0.9800 & $\mathrm{C} 38-\mathrm{H} 38 \mathrm{C}$ & 0.9700 \\
\hline $\mathrm{C} 11-\mathrm{H} 11 \mathrm{~B}$ & 0.9800 & C $38-\mathrm{H} 38 \mathrm{~B}$ & 0.9700 \\
\hline $\mathrm{C} 12-\mathrm{C} 17$ & $1.378(6)$ & $\mathrm{C} 38-\mathrm{H} 38 \mathrm{~A}$ & 0.9700 \\
\hline $\mathrm{C} 12-\mathrm{C} 13$ & $1.408(7)$ & $\mathrm{C} 40-\mathrm{Cl} 4$ & $1.625(12)$ \\
\hline $\mathrm{C} 13-\mathrm{C} 14$ & $1.370(9)$ & $\mathrm{C} 40-\mathrm{Cl} 3$ & $1.772(11)$ \\
\hline $\mathrm{C} 13-\mathrm{H} 13$ & 0.9400 & $\mathrm{C} 40-\mathrm{H} 40 \mathrm{~A}$ & 0.9800 \\
\hline $\mathrm{C} 14-\mathrm{C} 15$ & $1.343(9)$ & $\mathrm{C} 40-\mathrm{H} 40 \mathrm{~B}$ & 0.9800 \\
\hline $\mathrm{C} 14-\mathrm{H} 14$ & 0.9400 & & \\
\hline $\mathrm{N} 1-\mathrm{Zn} 1-\mathrm{N} 2$ & $75.02(12)$ & $\mathrm{C} 16-\mathrm{C} 15-\mathrm{H} 15$ & 121.0 \\
\hline $\mathrm{N} 1-\mathrm{Zn} 1-\mathrm{N} 5$ & $74.23(12)$ & $\mathrm{C} 17-\mathrm{C} 16-\mathrm{C} 15$ & $121.2(6)$ \\
\hline $\mathrm{N} 2-\mathrm{Zn} 1-\mathrm{N} 5$ & $149.21(11)$ & $\mathrm{C} 17-\mathrm{C} 16-\mathrm{H} 16$ & 119.4 \\
\hline $\mathrm{N} 1-\mathrm{Zn} 1-\mathrm{Cl} 1$ & $114.15(9)$ & $\mathrm{C} 15-\mathrm{C} 16-\mathrm{H} 16$ & 119.4 \\
\hline $\mathrm{N} 1-\mathrm{Zn} 1-\mathrm{Cl} 2$ & $114.68(9)$ & $\mathrm{C} 12-\mathrm{C} 17-\mathrm{C} 16$ & $120.4(6)$ \\
\hline $\mathrm{C} 11-\mathrm{Zn} 1-\mathrm{N} 2$ & $98.12(8)$ & $\mathrm{C} 12-\mathrm{C} 17-\mathrm{H} 17$ & 119.8 \\
\hline $\mathrm{Cl} 2-\mathrm{Zn} 1-\mathrm{N} 2$ & $95.70(9)$ & $\mathrm{C} 16-\mathrm{C} 17-\mathrm{H} 17$ & 119.8 \\
\hline $\mathrm{Cl1}-\mathrm{Zn} 1-\mathrm{N} 5$ & $93.02(7)$ & $\mathrm{N} 3-\mathrm{C} 18-\mathrm{H} 18 \mathrm{C}$ & 109.5 \\
\hline $\mathrm{Cl} 2-\mathrm{Zn} 1-\mathrm{N} 5$ & $98.34(8)$ & $\mathrm{N} 3-\mathrm{C} 18-\mathrm{H} 18 \mathrm{~B}$ & 109.5 \\
\hline $\mathrm{C} 11-\mathrm{Zn} 1-\mathrm{Cl} 2$ & $131.14(4)$ & $\mathrm{H} 18 \mathrm{C}-\mathrm{C} 18-\mathrm{H} 18 \mathrm{~B}$ & 109.5 \\
\hline $\mathrm{C} 21-\mathrm{N} 1-\mathrm{C} 1$ & $120.8(3)$ & $\mathrm{N} 3-\mathrm{C} 18-\mathrm{H} 18 \mathrm{~A}$ & 109.5 \\
\hline $\mathrm{C} 21-\mathrm{N} 1-\mathrm{Zn} 1$ & $120.7(2)$ & $\mathrm{H} 18 \mathrm{C}-\mathrm{C} 18-\mathrm{H} 18 \mathrm{~A}$ & 109.5 \\
\hline $\mathrm{C} 1-\mathrm{N} 1-\mathrm{Zn} 1$ & $118.3(3)$ & $\mathrm{H} 18 \mathrm{~B}-\mathrm{C} 18-\mathrm{H} 18 \mathrm{~A}$ & 109.5 \\
\hline $\mathrm{C} 4-\mathrm{N} 2-\mathrm{C} 3$ & $114.5(3)$ & $\mathrm{N} 1-\mathrm{C} 21-\mathrm{C} 22$ & $119.6(3)$ \\
\hline $\mathrm{C} 4-\mathrm{N} 2-\mathrm{C} 10$ & $111.2(3)$ & $\mathrm{N} 1-\mathrm{C} 21-\mathrm{C} 23$ & $115.2(3)$ \\
\hline $\mathrm{C} 3-\mathrm{N} 2-\mathrm{C} 10$ & $109.9(3)$ & $\mathrm{C} 22-\mathrm{C} 21-\mathrm{C} 23$ & $125.1(4)$ \\
\hline $\mathrm{C} 4-\mathrm{N} 2-\mathrm{Zn} 1$ & $110.2(2)$ & $\mathrm{N} 4-\mathrm{C} 22-\mathrm{C} 21$ & $119.8(4)$ \\
\hline $\mathrm{C} 3-\mathrm{N} 2-\mathrm{Zn} 1$ & $99.2(2)$ & $\mathrm{N} 4-\mathrm{C} 22-\mathrm{C} 31$ & $117.4(3)$ \\
\hline $\mathrm{C} 10-\mathrm{N} 2-\mathrm{Zn} 1$ & $111.3(3)$ & $\mathrm{C} 21-\mathrm{C} 22-\mathrm{C} 31$ & $122.8(4)$ \\
\hline $\mathrm{C} 12-\mathrm{N} 3-\mathrm{C} 18$ & $120.4(4)$ & $\mathrm{N} 5-\mathrm{C} 23-\mathrm{C} 21$ & $109.2(3)$ \\
\hline $\mathrm{C} 12-\mathrm{N} 3-\mathrm{C} 11$ & $120.1(4)$ & $\mathrm{N} 5-\mathrm{C} 23-\mathrm{H} 23 \mathrm{~A}$ & 109.8 \\
\hline $\mathrm{C} 18-\mathrm{N} 3-\mathrm{C} 11$ & $117.4(4)$ & $\mathrm{C} 21-\mathrm{C} 23-\mathrm{H} 23 \mathrm{~A}$ & 109.8 \\
\hline $\mathrm{C} 2-\mathrm{N} 4-\mathrm{C} 22$ & $119.8(3)$ & $\mathrm{N} 5-\mathrm{C} 23-\mathrm{H} 23 \mathrm{~B}$ & 109.8 \\
\hline $\mathrm{C} 24-\mathrm{N} 5-\mathrm{C} 30$ & $111.1(3)$ & $\mathrm{C} 21-\mathrm{C} 23-\mathrm{H} 23 \mathrm{~B}$ & 109.8 \\
\hline $\mathrm{C} 24-\mathrm{N} 5-\mathrm{C} 23$ & $114.7(3)$ & $\mathrm{H} 23 \mathrm{~A}-\mathrm{C} 23-\mathrm{H} 23 \mathrm{~B}$ & 108.3 \\
\hline $\mathrm{C} 30-\mathrm{N} 5-\mathrm{C} 23$ & $109.3(3)$ & $\mathrm{C} 25-\mathrm{C} 24-\mathrm{C} 29$ & $119.5(4)$ \\
\hline $\mathrm{C} 24-\mathrm{N} 5-\mathrm{Zn} 1$ & $109.4(2)$ & $\mathrm{C} 25-\mathrm{C} 24-\mathrm{N} 5$ & $123.5(4)$ \\
\hline $\mathrm{C} 30-\mathrm{N} 5-\mathrm{Zn} 1$ & $111.1(3)$ & $\mathrm{C} 29-\mathrm{C} 24-\mathrm{N} 5$ & $117.0(4)$ \\
\hline $\mathrm{C} 23-\mathrm{N} 5-\mathrm{Zn} 1$ & $100.9(2)$ & $\mathrm{C} 24-\mathrm{C} 25-\mathrm{C} 26$ & $119.4(4)$ \\
\hline $\mathrm{C} 32-\mathrm{N} 6-\mathrm{C} 31$ & $122.1(4)$ & $\mathrm{C} 24-\mathrm{C} 25-\mathrm{H} 25$ & 120.3 \\
\hline $\mathrm{C} 32-\mathrm{N} 6-\mathrm{C} 38$ & $122.1(3)$ & $\mathrm{C} 26-\mathrm{C} 25-\mathrm{H} 25$ & 120.3 \\
\hline $\mathrm{C} 31-\mathrm{N} 6-\mathrm{C} 38$ & $115.2(4)$ & $\mathrm{C} 27-\mathrm{C} 26-\mathrm{C} 25$ & $120.5(4)$ \\
\hline $\mathrm{N} 1-\mathrm{C} 1-\mathrm{C} 2$ & $118.9(4)$ & $\mathrm{C} 27-\mathrm{C} 26-\mathrm{H} 26$ & 119.7 \\
\hline $\mathrm{N} 1-\mathrm{C} 1-\mathrm{C} 3$ & $115.6(3)$ & $\mathrm{C} 25-\mathrm{C} 26-\mathrm{H} 26$ & 119.7 \\
\hline $\mathrm{C} 2-\mathrm{C} 1-\mathrm{C} 3$ & $125.4(4)$ & $\mathrm{C} 28-\mathrm{C} 27-\mathrm{C} 26$ & $120.2(4)$ \\
\hline $\mathrm{N} 4-\mathrm{C} 2-\mathrm{C} 1$ & $120.9(4)$ & $\mathrm{C} 28-\mathrm{C} 27-\mathrm{H} 27$ & 119.9 \\
\hline $\mathrm{N} 4-\mathrm{C} 2-\mathrm{C} 11$ & $118.2(3)$ & $\mathrm{C} 26-\mathrm{C} 27-\mathrm{H} 27$ & 119.9 \\
\hline
\end{tabular}




\begin{tabular}{|c|c|c|c|}
\hline $\mathrm{C} 1-\mathrm{C} 2-\mathrm{C} 11$ & $120.7(4)$ & $\mathrm{C} 27-\mathrm{C} 28-\mathrm{C} 29$ & $119.6(4)$ \\
\hline $\mathrm{N} 2-\mathrm{C} 3-\mathrm{C} 1$ & $110.9(3)$ & $\mathrm{C} 27-\mathrm{C} 28-\mathrm{H} 28$ & 120.2 \\
\hline $\mathrm{N} 2-\mathrm{C} 3-\mathrm{H} 3 \mathrm{~A}$ & 109.5 & $\mathrm{C} 29-\mathrm{C} 28-\mathrm{H} 28$ & 120.2 \\
\hline $\mathrm{C} 1-\mathrm{C} 3-\mathrm{H} 3 \mathrm{~A}$ & 109.5 & $\mathrm{C} 28-\mathrm{C} 29-\mathrm{C} 24$ & $120.7(4)$ \\
\hline $\mathrm{N} 2-\mathrm{C} 3-\mathrm{H} 3 \mathrm{~B}$ & 109.5 & $\mathrm{C} 28-\mathrm{C} 29-\mathrm{H} 29$ & 119.6 \\
\hline $\mathrm{C} 1-\mathrm{C} 3-\mathrm{H} 3 \mathrm{~B}$ & 109.5 & $\mathrm{C} 24-\mathrm{C} 29-\mathrm{H} 29$ & 119.6 \\
\hline $\mathrm{H} 3 \mathrm{~A}-\mathrm{C} 3-\mathrm{H} 3 \mathrm{~B}$ & 108.1 & $\mathrm{~N} 5-\mathrm{C} 30-\mathrm{H} 30 \mathrm{C}$ & 109.5 \\
\hline $\mathrm{C} 5-\mathrm{C} 4-\mathrm{C} 9$ & $118.8(4)$ & $\mathrm{N} 5-\mathrm{C} 30-\mathrm{H} 30 \mathrm{~B}$ & 109.5 \\
\hline $\mathrm{C} 5-\mathrm{C} 4-\mathrm{N} 2$ & $123.3(4)$ & $\mathrm{H} 30 \mathrm{C}-\mathrm{C} 30-\mathrm{H} 30 \mathrm{~B}$ & 109.5 \\
\hline $\mathrm{C} 9-\mathrm{C} 4-\mathrm{N} 2$ & $117.8(4)$ & $\mathrm{N} 5-\mathrm{C} 30-\mathrm{H} 30 \mathrm{~A}$ & 109.5 \\
\hline $\mathrm{C} 6-\mathrm{C} 5-\mathrm{C} 4$ & $120.0(4)$ & $\mathrm{H} 30 \mathrm{C}-\mathrm{C} 30-\mathrm{H} 30 \mathrm{~A}$ & 109.5 \\
\hline $\mathrm{C} 6-\mathrm{C} 5-\mathrm{H} 5$ & 120.0 & $\mathrm{H} 30 \mathrm{~B}-\mathrm{C} 30-\mathrm{H} 30 \mathrm{~A}$ & 109.5 \\
\hline $\mathrm{C} 4-\mathrm{C} 5-\mathrm{H} 5$ & 120.0 & $\mathrm{~N} 6-\mathrm{C} 31-\mathrm{C} 22$ & $114.2(4)$ \\
\hline $\mathrm{C} 7-\mathrm{C} 6-\mathrm{C} 5$ & $121.1(5)$ & $\mathrm{N} 6-\mathrm{C} 31-\mathrm{H} 31 \mathrm{~A}$ & 108.7 \\
\hline $\mathrm{C} 7-\mathrm{C} 6-\mathrm{H} 6$ & 119.5 & $\mathrm{C} 22-\mathrm{C} 31-\mathrm{H} 31 \mathrm{~A}$ & 108.7 \\
\hline $\mathrm{C} 5-\mathrm{C} 6-\mathrm{H} 6$ & 119.5 & $\mathrm{~N} 6-\mathrm{C} 31-\mathrm{H} 31 \mathrm{~B}$ & 108.7 \\
\hline $\mathrm{C} 6-\mathrm{C} 7-\mathrm{C} 8$ & $119.4(5)$ & $\mathrm{C} 22-\mathrm{C} 31-\mathrm{H} 31 \mathrm{~B}$ & 108.7 \\
\hline $\mathrm{C} 6-\mathrm{C} 7-\mathrm{H} 7$ & 120.3 & $\mathrm{H} 31 \mathrm{~A}-\mathrm{C} 31-\mathrm{H} 31 \mathrm{~B}$ & 107.6 \\
\hline $\mathrm{C} 8-\mathrm{C} 7-\mathrm{H} 7$ & 120.3 & $\mathrm{~N} 6-\mathrm{C} 32-\mathrm{C} 37$ & $122.3(4)$ \\
\hline $\mathrm{C} 7-\mathrm{C} 8-\mathrm{C} 9$ & $120.5(5)$ & $\mathrm{N} 6-\mathrm{C} 32-\mathrm{C} 33$ & $120.4(4)$ \\
\hline $\mathrm{C} 7-\mathrm{C} 8-\mathrm{H} 8$ & 119.8 & $\mathrm{C} 37-\mathrm{C} 32-\mathrm{C} 33$ & $117.3(4)$ \\
\hline $\mathrm{C} 9-\mathrm{C} 8-\mathrm{H} 8$ & 119.8 & $\mathrm{C} 34-\mathrm{C} 33-\mathrm{C} 32$ & $119.6(5)$ \\
\hline $\mathrm{C} 8-\mathrm{C} 9-\mathrm{C} 4$ & $120.3(5)$ & $\mathrm{C} 34-\mathrm{C} 33-\mathrm{H} 33$ & 120.2 \\
\hline $\mathrm{C} 8-\mathrm{C} 9-\mathrm{H} 9$ & 119.9 & $\mathrm{C} 32-\mathrm{C} 33-\mathrm{H} 33$ & 120.2 \\
\hline $\mathrm{C} 4-\mathrm{C} 9-\mathrm{H} 9$ & 119.9 & $\mathrm{C} 35-\mathrm{C} 34-\mathrm{C} 33$ & $122.9(5)$ \\
\hline $\mathrm{N} 2-\mathrm{C} 10-\mathrm{H} 10 \mathrm{C}$ & 109.5 & $\mathrm{C} 35-\mathrm{C} 34-\mathrm{H} 34$ & 118.5 \\
\hline $\mathrm{N} 2-\mathrm{C} 10-\mathrm{H} 10 \mathrm{~B}$ & 109.5 & $\mathrm{C} 33-\mathrm{C} 34-\mathrm{H} 34$ & 118.5 \\
\hline $\mathrm{H} 10 \mathrm{C}-\mathrm{C} 10-\mathrm{H} 10 \mathrm{~B}$ & 109.5 & $\mathrm{C} 34-\mathrm{C} 35-\mathrm{C} 36$ & $117.6(5)$ \\
\hline $\mathrm{N} 2-\mathrm{C} 10-\mathrm{H} 10 \mathrm{~A}$ & 109.5 & $\mathrm{C} 34-\mathrm{C} 35-\mathrm{H} 35$ & 121.2 \\
\hline $\mathrm{H} 10 \mathrm{C}-\mathrm{C} 10-\mathrm{H} 10 \mathrm{~A}$ & 109.5 & $\mathrm{C} 36-\mathrm{C} 35-\mathrm{H} 35$ & 121.2 \\
\hline $\mathrm{H} 10 \mathrm{~B}-\mathrm{C} 10-\mathrm{H} 10 \mathrm{~A}$ & 109.5 & $\mathrm{C} 37-\mathrm{C} 36-\mathrm{C} 35$ & $120.6(5)$ \\
\hline $\mathrm{N} 3-\mathrm{C} 11-\mathrm{C} 2$ & $114.2(4)$ & $\mathrm{C} 37-\mathrm{C} 36-\mathrm{H} 36$ & 119.7 \\
\hline N3-C11-H11A & 108.7 & $\mathrm{C} 35-\mathrm{C} 36-\mathrm{H} 36$ & 119.7 \\
\hline $\mathrm{C} 2-\mathrm{C} 11-\mathrm{H} 11 \mathrm{~A}$ & 108.7 & $\mathrm{C} 36-\mathrm{C} 37-\mathrm{C} 32$ & $121.9(5)$ \\
\hline N3-C11-H11B & 108.7 & $\mathrm{C} 36-\mathrm{C} 37-\mathrm{H} 37$ & 119.1 \\
\hline $\mathrm{C} 2-\mathrm{C} 11-\mathrm{H} 11 \mathrm{~B}$ & 108.7 & $\mathrm{C} 32-\mathrm{C} 37-\mathrm{H} 37$ & 119.1 \\
\hline $\mathrm{H} 11 \mathrm{~A}-\mathrm{C} 11-\mathrm{H} 11 \mathrm{~B}$ & 107.6 & $\mathrm{~N} 6-\mathrm{C} 38-\mathrm{H} 38 \mathrm{C}$ & 109.5 \\
\hline $\mathrm{C} 17-\mathrm{C} 12-\mathrm{N} 3$ & 122.3 & N6-C38-H38B & 109.5 \\
\hline $\mathrm{C} 17-\mathrm{C} 12-\mathrm{C} 13$ & $117.4(5)$ & $\mathrm{H} 38 \mathrm{C}-\mathrm{C} 38-\mathrm{H} 38 \mathrm{~B}$ & 109.5 \\
\hline $\mathrm{N} 3-\mathrm{C} 12-\mathrm{C} 13$ & 120.3 & $\mathrm{~N} 6-\mathrm{C} 38-\mathrm{H} 38 \mathrm{~A}$ & 109.5 \\
\hline $\mathrm{C} 14-\mathrm{C} 13-\mathrm{C} 12$ & $120.5(6)$ & $\mathrm{H} 38 \mathrm{C}-\mathrm{C} 38-\mathrm{H} 38 \mathrm{~A}$ & 109.5 \\
\hline $\mathrm{C} 14-\mathrm{C} 13-\mathrm{H} 13$ & 119.8 & $\mathrm{H} 38 \mathrm{~B}-\mathrm{C} 38-\mathrm{H} 38 \mathrm{~A}$ & 109.5 \\
\hline $\mathrm{C} 12-\mathrm{C} 13-\mathrm{H} 13$ & 119.8 & $\mathrm{Cl} 4-\mathrm{C} 40-\mathrm{Cl} 3$ & $118.1(6)$ \\
\hline $\mathrm{C} 15-\mathrm{C} 14-\mathrm{C} 13$ & $122.5(6)$ & $\mathrm{Cl} 4-\mathrm{C} 40-\mathrm{H} 40 \mathrm{~A}$ & 107.8 \\
\hline $\mathrm{C} 15-\mathrm{C} 14-\mathrm{H} 14$ & 118.8 & $\mathrm{Cl} 3-\mathrm{C} 40-\mathrm{H} 40 \mathrm{~A}$ & 107.8 \\
\hline $\mathrm{C} 13-\mathrm{C} 14-\mathrm{H} 14$ & 118.8 & $\mathrm{Cl} 4-\mathrm{C} 40-\mathrm{H} 40 \mathrm{~B}$ & 107.8 \\
\hline $\mathrm{C} 14-\mathrm{C} 15-\mathrm{C} 16$ & $118.0(6)$ & $\mathrm{Cl} 3-\mathrm{C} 40-\mathrm{H} 40 \mathrm{~B}$ & 107.8 \\
\hline $\mathrm{C} 14-\mathrm{C} 15-\mathrm{H} 15$ & 121.0 & $\mathrm{H} 40 \mathrm{~A}-\mathrm{C} 40-\mathrm{H} 40 \mathrm{~B}$ & 107.1 \\
\hline
\end{tabular}




\begin{tabular}{|c|c|c|c|}
\hline $\mathrm{C} 21-\mathrm{N} 1-\mathrm{C} 1-\mathrm{C} 2$ & $0.9(5)$ & $\mathrm{C} 1-\mathrm{N} 1-\mathrm{C} 21-\mathrm{C} 22$ & $0.8(5)$ \\
\hline $\mathrm{Zn} 1-\mathrm{N} 1-\mathrm{C} 1-\mathrm{C} 2$ & $-173.8(3)$ & $\mathrm{Zn} 1-\mathrm{N} 1-\mathrm{C} 21-\mathrm{C} 22$ & $175.4(3)$ \\
\hline $\mathrm{C} 21-\mathrm{N} 1-\mathrm{C} 1-\mathrm{C} 3$ & $177.6(3)$ & $\mathrm{C} 1-\mathrm{N} 1-\mathrm{C} 21-\mathrm{C} 23$ & $179.5(3)$ \\
\hline $\mathrm{Zn} 1-\mathrm{N} 1-\mathrm{C} 1-\mathrm{C} 3$ & $2.8(4)$ & $\mathrm{Zn} 1-\mathrm{N} 1-\mathrm{C} 21-\mathrm{C} 23$ & $-5.9(4)$ \\
\hline $\mathrm{C} 22-\mathrm{N} 4-\mathrm{C} 2-\mathrm{C} 1$ & $0.6(6)$ & $\mathrm{C} 2-\mathrm{N} 4-\mathrm{C} 22-\mathrm{C} 21$ & $1.2(6)$ \\
\hline $\mathrm{C} 22-\mathrm{N} 4-\mathrm{C} 2-\mathrm{C} 11$ & $-175.4(4)$ & $\mathrm{C} 2-\mathrm{N} 4-\mathrm{C} 22-\mathrm{C} 31$ & $-178.7(4)$ \\
\hline $\mathrm{N} 1-\mathrm{C} 1-\mathrm{C} 2-\mathrm{N} 4$ & $-1.7(6)$ & $\mathrm{N} 1-\mathrm{C} 21-\mathrm{C} 22-\mathrm{N} 4$ & $-1.9(5)$ \\
\hline $\mathrm{C} 3-\mathrm{C} 1-\mathrm{C} 2-\mathrm{N} 4$ & $-178.0(4)$ & $\mathrm{C} 23-\mathrm{C} 21-\mathrm{C} 22-\mathrm{N} 4$ & $179.6(3)$ \\
\hline $\mathrm{N} 1-\mathrm{C} 1-\mathrm{C} 2-\mathrm{C} 11$ & $174.2(4)$ & $\mathrm{N} 1-\mathrm{C} 21-\mathrm{C} 22-\mathrm{C} 31$ & $178.0(4)$ \\
\hline $\mathrm{C} 3-\mathrm{C} 1-\mathrm{C} 2-\mathrm{C} 11$ & $-2.0(6)$ & $\mathrm{C} 23-\mathrm{C} 21-\mathrm{C} 22-\mathrm{C} 31$ & $-0.5(6)$ \\
\hline $\mathrm{C} 4-\mathrm{N} 2-\mathrm{C} 3-\mathrm{C} 1$ & $-163.3(3)$ & $\mathrm{C} 24-\mathrm{N} 5-\mathrm{C} 23-\mathrm{C} 21$ & $-162.9(3)$ \\
\hline $\mathrm{C} 10-\mathrm{N} 2-\mathrm{C} 3-\mathrm{C} 1$ & $70.8(4)$ & $\mathrm{C} 30-\mathrm{N} 5-\mathrm{C} 23-\mathrm{C} 21$ & $71.6(4)$ \\
\hline $\mathrm{Zn} 1-\mathrm{N} 2-\mathrm{C} 3-\mathrm{C} 1$ & $-46.0(3)$ & $\mathrm{Zn} 1-\mathrm{N} 5-\mathrm{C} 23-\mathrm{C} 21$ & $-45.5(3)$ \\
\hline $\mathrm{N} 1-\mathrm{C} 1-\mathrm{C} 3-\mathrm{N} 2$ & $34.4(5)$ & $\mathrm{N} 1-\mathrm{C} 21-\mathrm{C} 23-\mathrm{N} 5$ & $39.1(4)$ \\
\hline $\mathrm{C} 2-\mathrm{C} 1-\mathrm{C} 3-\mathrm{N} 2$ & $-149.2(4)$ & $\mathrm{C} 22-\mathrm{C} 21-\mathrm{C} 23-\mathrm{N} 5$ & $-142.4(4)$ \\
\hline $\mathrm{C} 3-\mathrm{N} 2-\mathrm{C} 4-\mathrm{C} 5$ & $-7.8(6)$ & $\mathrm{C} 30-\mathrm{N} 5-\mathrm{C} 24-\mathrm{C} 25$ & $112.7(4)$ \\
\hline $\mathrm{C} 10-\mathrm{N} 2-\mathrm{C} 4-\mathrm{C} 5$ & $117.5(4)$ & $\mathrm{C} 23-\mathrm{N} 5-\mathrm{C} 24-\mathrm{C} 25$ & $-11.8(5)$ \\
\hline $\mathrm{Zn} 1-\mathrm{N} 2-\mathrm{C} 4-\mathrm{C} 5$ & $-118.6(4)$ & $\mathrm{Zn} 1-\mathrm{N} 5-\mathrm{C} 24-\mathrm{C} 25$ & $-124.3(3)$ \\
\hline $\mathrm{C} 3-\mathrm{N} 2-\mathrm{C} 4-\mathrm{C} 9$ & $171.3(4)$ & $\mathrm{C} 30-\mathrm{N} 5-\mathrm{C} 24-\mathrm{C} 29$ & $-68.9(5)$ \\
\hline $\mathrm{C} 10-\mathrm{N} 2-\mathrm{C} 4-\mathrm{C} 9$ & $-63.5(5)$ & $\mathrm{C} 23-\mathrm{N} 5-\mathrm{C} 24-\mathrm{C} 29$ & $166.6(3)$ \\
\hline $\mathrm{Zn} 1-\mathrm{N} 2-\mathrm{C} 4-\mathrm{C} 9$ & $60.4(4)$ & $\mathrm{Zn} 1-\mathrm{N} 5-\mathrm{C} 24-\mathrm{C} 29$ & $54.1(4)$ \\
\hline $\mathrm{C} 9-\mathrm{C} 4-\mathrm{C} 5-\mathrm{C} 6$ & $-2.2(6)$ & $\mathrm{C} 29-\mathrm{C} 24-\mathrm{C} 25-\mathrm{C} 26$ & $-0.5(6)$ \\
\hline $\mathrm{N} 2-\mathrm{C} 4-\mathrm{C} 5-\mathrm{C} 6$ & $176.9(4)$ & $\mathrm{N} 5-\mathrm{C} 24-\mathrm{C} 25-\mathrm{C} 26$ & $177.9(4)$ \\
\hline $\mathrm{C} 4-\mathrm{C} 5-\mathrm{C} 6-\mathrm{C} 7$ & $1.9(7)$ & $\mathrm{C} 24-\mathrm{C} 25-\mathrm{C} 26-\mathrm{C} 27$ & $1.1(6)$ \\
\hline $\mathrm{C} 5-\mathrm{C} 6-\mathrm{C} 7-\mathrm{C} 8$ & $-0.6(7)$ & $\mathrm{C} 25-\mathrm{C} 26-\mathrm{C} 27-\mathrm{C} 28$ & $-0.8(7)$ \\
\hline $\mathrm{C} 6-\mathrm{C} 7-\mathrm{C} 8-\mathrm{C} 9$ & $-0.3(8)$ & $\mathrm{C} 26-\mathrm{C} 27-\mathrm{C} 28-\mathrm{C} 29$ & $-0.1(7)$ \\
\hline $\mathrm{C} 7-\mathrm{C} 8-\mathrm{C} 9-\mathrm{C} 4$ & $0.0(8)$ & $\mathrm{C} 27-\mathrm{C} 28-\mathrm{C} 29-\mathrm{C} 24$ & $0.7(7)$ \\
\hline $\mathrm{C} 5-\mathrm{C} 4-\mathrm{C} 9-\mathrm{C} 8$ & $1.2(7)$ & $\mathrm{C} 25-\mathrm{C} 24-\mathrm{C} 29-\mathrm{C} 28$ & $-0.3(6)$ \\
\hline $\mathrm{N} 2-\mathrm{C} 4-\mathrm{C} 9-\mathrm{C} 8$ & $-177.9(4)$ & $\mathrm{N} 5-\mathrm{C} 24-\mathrm{C} 29-\mathrm{C} 28$ & $-178.9(4)$ \\
\hline $\mathrm{C} 12-\mathrm{N} 3-\mathrm{C} 11-\mathrm{C} 2$ & $-80.2(5)$ & $\mathrm{C} 32-\mathrm{N} 6-\mathrm{C} 31-\mathrm{C} 22$ & $99.2(5)$ \\
\hline $\mathrm{C} 18-\mathrm{N} 3-\mathrm{C} 11-\mathrm{C} 2$ & $83.5(5)$ & $\mathrm{C} 38-\mathrm{N} 6-\mathrm{C} 31-\mathrm{C} 22$ & $-72.1(5)$ \\
\hline $\mathrm{N} 4-\mathrm{C} 2-\mathrm{C} 11-\mathrm{N} 3$ & $-8.2(6)$ & $\mathrm{N} 4-\mathrm{C} 22-\mathrm{C} 31-\mathrm{N} 6$ & $-30.4(6)$ \\
\hline $\mathrm{C} 1-\mathrm{C} 2-\mathrm{C} 11-\mathrm{N} 3$ & $175.7(4)$ & $\mathrm{C} 21-\mathrm{C} 22-\mathrm{C} 31-\mathrm{N} 6$ & $149.7(4)$ \\
\hline $\mathrm{C} 18-\mathrm{N} 3-\mathrm{C} 12-\mathrm{C} 17$ & $-163.8(5)$ & $\mathrm{C} 31-\mathrm{N} 6-\mathrm{C} 32-\mathrm{C} 37$ & $-13.3(7)$ \\
\hline $\mathrm{C} 11-\mathrm{N} 3-\mathrm{C} 12-\mathrm{C} 17$ & $-0.6(7)$ & $\mathrm{C} 38-\mathrm{N} 6-\mathrm{C} 32-\mathrm{C} 37$ & $157.3(5)$ \\
\hline $\mathrm{C} 18-\mathrm{N} 3-\mathrm{C} 12-\mathrm{C} 13$ & $18.0(7)$ & $\mathrm{C} 31-\mathrm{N} 6-\mathrm{C} 32-\mathrm{C} 33$ & $164.9(4)$ \\
\hline $\mathrm{C} 11-\mathrm{N} 3-\mathrm{C} 12-\mathrm{C} 13$ & $-178.8(4)$ & $\mathrm{C} 38-\mathrm{N} 6-\mathrm{C} 32-\mathrm{C} 33$ & $-24.5(7)$ \\
\hline $\mathrm{C} 17-\mathrm{C} 12-\mathrm{C} 13-\mathrm{C} 14$ & $0.8(8)$ & $\mathrm{N} 6-\mathrm{C} 32-\mathrm{C} 33-\mathrm{C} 34$ & $-176.1(5)$ \\
\hline $\mathrm{N} 3-\mathrm{C} 12-\mathrm{C} 13-\mathrm{C} 14$ & $179.1(5)$ & $\mathrm{C} 37-\mathrm{C} 32-\mathrm{C} 33-\mathrm{C} 34$ & $2.2(7)$ \\
\hline $\mathrm{C} 12-\mathrm{C} 13-\mathrm{C} 14-\mathrm{C} 15$ & $-0.4(10)$ & $\mathrm{C} 32-\mathrm{C} 33-\mathrm{C} 34-\mathrm{C} 35$ & $-0.5(9)$ \\
\hline $\mathrm{C} 13-\mathrm{C} 14-\mathrm{C} 15-\mathrm{C} 16$ & $0.0(10)$ & $\mathrm{C} 33-\mathrm{C} 34-\mathrm{C} 35-\mathrm{C} 36$ & $-1.8(9)$ \\
\hline $\mathrm{C} 14-\mathrm{C} 15-\mathrm{C} 16-\mathrm{C} 17$ & $0.0(9)$ & $\mathrm{C} 34-\mathrm{C} 35-\mathrm{C} 36-\mathrm{C} 37$ & $2.3(9)$ \\
\hline $\mathrm{N} 3-\mathrm{C} 12-\mathrm{C} 17-\mathrm{C} 16$ & $-179.1(5)$ & $\mathrm{C} 35-\mathrm{C} 36-\mathrm{C} 37-\mathrm{C} 32$ & $-0.7(8)$ \\
\hline $\mathrm{C} 13-\mathrm{C} 12-\mathrm{C} 17-\mathrm{C} 16$ & $-0.8(7)$ & $\mathrm{N} 6-\mathrm{C} 32-\mathrm{C} 37-\mathrm{C} 36$ & $176.7(5)$ \\
\hline $\mathrm{C} 15-\mathrm{C} 16-\mathrm{C} 17-\mathrm{C} 12$ & $0.4(8)$ & $\mathrm{C} 33-\mathrm{C} 32-\mathrm{C} 37-\mathrm{C} 36$ & $-1.6(7)$ \\
\hline
\end{tabular}




\section{supporting information}

Hydrogen-bond geometry $\left(\AA,{ }^{\circ}\right)$

$\mathrm{Cg} 3$ is the centroid of the pyrazine ring $\mathrm{N} 1 / \mathrm{N} 4 / \mathrm{C} 1 / \mathrm{C} 2 / \mathrm{C} 21 / \mathrm{C} 22$, and $\mathrm{Cg} 5$ and $\mathrm{Cg} 7$ are the centroids of rings $\mathrm{C} 12-\mathrm{C} 17$ and $\mathrm{C} 32-\mathrm{C} 37$, respectively.

\begin{tabular}{lllll}
\hline$D-\mathrm{H} \cdots A$ & $D-\mathrm{H}$ & $\mathrm{H} \cdots A$ & $D \cdots A$ & $D-\mathrm{H} \cdots A$ \\
\hline $\mathrm{C} 6-\mathrm{H} 6 \cdots \mathrm{Cg} 7^{\mathrm{i}}$ & 0.94 & 2.88 & $3.814(6)$ & 177 \\
$\mathrm{C} 11-\mathrm{H} 11 B \cdots \mathrm{Cg} 5^{\mathrm{ii}}$ & 0.98 & 2.90 & $3.540(5)$ & 124 \\
$\mathrm{C} 26-\mathrm{H} 26 \cdots \mathrm{Cg} 3^{\mathrm{iii}}$ & 0.94 & 2.95 & $3.544(5)$ & 122 \\
$\mathrm{Zn} 1-\mathrm{Cl} 2 \cdots \mathrm{Cg} 3^{\text {iv }}$ & $2.24(1)$ & $3.68(1)$ & $5.8035(19)$ & $156(1)$
\end{tabular}

Symmetry codes: (i) $x-1, y-1, z$; (ii) $-x+1,-y,-z$; (iii) $-x+1,-y+1,-z+1$; (iv) $-x+1,-y,-z+1$. 